\title{
Lower hybrid resonances stimulated by the four CLUSTER relaxation sounders deep inside the plasmasphere: observations and inferred plasma characteristics
}

\author{
S. Kougblénou ${ }^{1}$, G. Lointier ${ }^{1}$, P. M. E. Décréau ${ }^{1}$, J.-G. Trotignon ${ }^{1}$, J.-L. Rauch ${ }^{1}$, X. Vallières ${ }^{1}$, P. Canu ${ }^{2}$, A. Masson ${ }^{3}$, \\ and J. Pickett ${ }^{4}$ \\ ${ }^{1}$ Laboratoire de Physique et Chimie de l'Environnement et de l'Espace (LPC2E), UMR6115, Orléans, France \\ ${ }^{2}$ Laboratoire de Physique des Plasmas (LPP), Palaiseau, France \\ ${ }^{3}$ ESA/ESTEC, Noordwijk, The Netherlands \\ ${ }^{4}$ University of Iowa, Iowa City, USA
}

Received: 19 April 2011 - Revised: 11 August 2011 - Accepted: 28 September 2011 - Published: 9 November 2011

\begin{abstract}
The frequency range of the WHISPER relaxation sounder instrument on board CLUSTER, 4-80 kHz, has been chosen so as to encompass the electron gyro-frequency, $F_{\mathrm{ce}}$, and the electron plasma frequency, $F_{\mathrm{p}}$, in most regions to be explored. Measurement of those frequencies, which are triggered as resonances by the sounder, provides a direct estimation of in situ fundamental plasma characteristics: electron density and magnetic field intensity. In the late mission phase, CLUSTER penetrated regions deep inside the plasmasphere where $F_{\text {ce }}$ and $F_{\mathrm{p}}$ are much higher than the upper frequency of the sounder's range. However, they are of the right order of magnitude as to place the lower hybrid frequency, $F_{\mathrm{lh}}$, in the $4-15 \mathrm{kHz}$ band. This characteristic frequency, placed at a resonance of the medium, is triggered by the sounder's transmitter and shows up as an isolated peak in the received spectrum, not present in spectra of naturally occuring VLF waves. This paper illustrates, from analysis of case events, how measured $F_{\mathrm{lh}}$ values give access to a plasma diagnostic novel of its kind. CLUSTER, travelling along its orbit, encounters favourable conditions where $F_{\mathrm{ce}}$ is increasing and $F_{\mathrm{p}}$ decreasing, such that $F_{\mathrm{ce}} / F_{\mathrm{p}}$ increases from values below unity to values above unity. Measured $F_{\mathrm{lh}}$ values thus give access, in turn, to the effective mass, $M_{\text {eff }}$, indicative of plasma ion composition, and to the core plasmasphere electron density value, a parameter difficult to measure. The analysed case events indicate that the estimated quantities $\left(M_{\text {eff }}\right.$ in the 1.0-1.4 range, $N_{\mathrm{e}}$ in the $5 \times 10^{2}-$ $10^{4} \mathrm{~cm}^{-3}$ range) are varying with external factors (altitude, $\mathrm{L}$ value, geomagnetic activity) in a plausible way. Although covering only a restricted region (mid-latitude, low altitude inner plasmasphere), these measurements are available, since
\end{abstract}

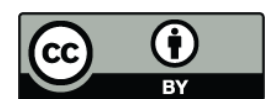

Correspondence to: S. Kougblénou (sena.kougblenou@cnrs-orleans.fr) late 2009, for all CLUSTER perigee passes not affected by eclipses (on average, roughly a third of a total of $\sim 200$ passes per year) and offer multipoint observations previously unavailable in this region.

Keywords. Magnetospheric physics (Plasmasphere; Plasma waves and instabilities)

\section{Introduction}

The WHISPER relaxation sounder instrument on board CLUSTER is designed both to monitor natural waves and to trigger plasma resonances in the so called high frequency range, in order to measure electron plasma density, $N_{\mathrm{e}}$, in the magnetosphere and near solar wind (Trotignon et al., 2001). The high frequency domain is controlled by electron motion (under respectively magnetic forces and electrostatic attraction from ions), in contrast with the low frequency domain (orders of magnitude lower), related to ion motions (of higher complexity, by token of a higher number of species). Characteristic resonances in the high frequency range are organized according to the plasma frequency $F_{\mathrm{p}}$, which varies as the square root of $N_{\mathrm{e}}$, and the gyro-frequency $F_{\text {ce }}$, which is proportional to the intensity of the magnetic field, $B_{0}$. The sounder's working frequency range is tuned to cover in particular the upper hybrid frequency $F_{\mathrm{uh}}\left(F_{\mathrm{uh}}=\right.$ $\left.\left(F_{\mathrm{p}}^{2}+F_{\mathrm{ce}}^{2}\right)^{1 / 2}\right)$, a frequency which is found near and above $F_{\mathrm{p}}$ or $F_{\mathrm{ce}}$, whichever is the largest. In the intermediate frequency range, one finds waves related to the lower hybrid characteristic frequency, $F_{\mathrm{lh}}$, driven by coupling of motions of ions and electrons. Its value, $F_{\mathrm{lh}}<\sim F_{\mathrm{ce}} / \sqrt{1836}$, is governed by the magnetic field intensity and ion total and relative concentrations.

Published by Copernicus Publications on behalf of the European Geosciences Union. 
The choice of an adequate working frequency range for WHISPER has been dictated by empirical knowledge of plasma frequency and gyro-frequency variations along the expected CLUSTER orbit, characterized by a high eccentricity (perigee $4.0 R_{\mathrm{E}}$, apogee $19.7 R_{\mathrm{E}}$, period $57 \mathrm{~h}$ ), a high inclination angle, and a major axis close to the ecliptic. Over the course of a year, CLUSTER apogee explores all local time sectors of geospace, from day side (solar wind upstream of the bow shock nose) to night side (central plasma sheet). In practice, the $F_{\mathrm{p}} / F_{\text {ce }}$ ratio is greater than one in all these regions, except sometimes near perigee. Other considerations have constrained the choice of frequency range, such as an adequate time and frequency resolution combined with limited telemetry rate, power and mass. The final design of the instrument (Décréau et al., 1997) is a compromise where the sounder working frequency is chosen inside the $4-80 \mathrm{kHz}$ range, and where natural emissions are analyzed in the range $2-80 \mathrm{kHz}$.

Once fixed, the instrumental frequency range defines the volume of magnetosphere explored by CLUSTER, which is actually accessible to WHISPER density diagnostic. The regions of highest priority regarding CLUSTER scientific objectives, i.e. cusp and magnetosheath (Escoubet et al., 1997), are well covered. Regions not always accessible to WHISPER density diagnostics include those with very low densities on the one hand, such as the magnetotail plasma lobes (when $N_{\mathrm{e}}<0.2 \mathrm{~cm}^{-3}$ ), and, on the other hand, those with high densities (when $N_{\mathrm{e}}>80 \mathrm{~cm}^{-3}$ ). The high density threshold is reached exceptionally in the (compressed) magnetosheath, and more often in the plasmasphere, near perigee. The Earth's plasmasphere region consists of cold plasma, escaped from the ionosphere, surrounding the Earth. Its outer envelope, the plasmasphere boundary layer (Carpenter and Lemaire, 2004), is roughly aligned with magnetic L shells. It is characterized by a sharp spatial gradient in density, defined in the reference study of Carpenter and Anderson (1992) as "a drop (of density value) by a factor of 5 or more occurring within $\Delta L=0.5$ ". The abovementioned study indicates that density profiles versus $L$ in the plasmapause layer generally includes WHISPER's density threshold, $N_{\mathrm{e}}=80 \mathrm{~cm}^{-3}$. Placed on average near $L=4$, the plasmapause extends in many cases to higher $L$ shells, above CLUSTER perigee near the equator. The orbit element placed below that boundary may thus be exposed to high $\left(>80 \mathrm{~cm}^{-3}\right)$ densities. For convenience, and when required for clarity, we shall coin regions characterized by densities above the $80 \mathrm{~cm}^{-3}$ threshold as "inner plasmasphere", and call "outer plasmasphere" regions those inside the plasmapause and characterized by densities below $80 \mathrm{~cm}^{-3}$. The inner plasmasphere is encountered near CLUSTER perigee on average once every two orbits during the early mission phase.

As years passed, the CLUSTER satellites plunged deeper inside the inner plasmasphere, such that plasma frequencies reached high values at each perigee pass, far above
WHISPER's instrumental capabilities (WHISPER frequency range still encompassing natural waves of electromagnetic nature, delivering a passive radiography of the inner plasmasphere). Since those regions, placed in the vicinity of the radiation belts, are forbidden for particle instrument operation, this meant a terra incognita as concerns in situ density estimation. However, as the CLUSTER orbits plunged still deeper inside the plasmasphere (at altitudes below $5000 \mathrm{~km}$ ) in 2009 and beyond, the sounder lower frequency limit moved below the domain of the lower hybrid resonance $F_{\mathrm{lh}}$, bringing at the same time the good news of very clear and well defined stimulated $F_{\mathrm{lh}}$ resonances.

First observations of lower hybrid resonance emissions in satellite data are presented in two grouped communications (Brice and Smith, 1964; Brice et al., 1964). The identification of the emissions is developed in Brice and Smith (1965). They analyse and discuss enhanced noise bands occurring naturally, observed in the range $\sim 4-10 \mathrm{kHz}$ by the satellite Alouette I moving in a very nearly circular orbit at an altitude of about $1000 \mathrm{~km}$. The frequency of the lower edge of those emissions is the local lower hybrid resonance $F_{\mathrm{lh}}$. Associated pioneer works were published during the same period. Smith and Brice (1964), examine the problem of propagation in any multi-component plasma, and Barrington et al. (1965), present results derived from the analysis of the Alouette-1 data. In the latter paper, the measured frequency $F_{\mathrm{lh}}$ is related to simultaneous measurements of the electron plasma frequency by the topside sounder in order to estimate ion composition and temperature of the ionosphere under various conditions. Similar and complementary observations have been obtained on board the Isis-2 satellite (Palmer and Barrington, 1973), indicating in particular that banded emissions above $F_{\mathrm{lh}}$ are enhanced when the sounder is in transmitting mode (at fixed, $1 \mathrm{MHz}$, frequency). Later, theoretical work in the frame of warm plasma theory near the $F_{\mathrm{lh}}$ resonance has been applied to Isis-2 observations (Hamelin, 1980), yielding a reasonable explanation of the duration of VLF echoes excited by the Isis 2 transmitter. More recent satellite missions reported observations of waves related to the local $F_{\mathrm{lh}}$ resonance, and discussed their generation, in particular by excitation via ordinary electromagnetic whistler-mode waves, both of natural and man-made origin (Bell et al., 1991, and references therein; Shklyar et al., 2010). Recently, $F_{\text {lh }}$ has been determined from the cut-offs of whistler-mode echoes of sounder-transmitted waves by the Radio Plasma Imager (RPI) (Reinish et al., 2000) on the IMAGE satellite when RPI is sounding from 6 to $63 \mathrm{kHz}$ (Sonwalkar et al., 2008; see plasmagrams in Fig. 5, Sonwalkar et al., 2011a).

WHISPER is, to our knowledge, the first relaxation sounder to trigger resonances at $F_{\mathrm{lh}}$. This confirms what could be anticipated from RPI observations. Compared to RPI (and to topside sounders), the relaxation technique, less demanding in power, concentrates on stimulating a small range of space (of order of $1 \mathrm{~km}$ ) around the satellite. Resonances are analyzed with good frequency resolution (see 
description in Sect. 2.1 above), hence leading to a truly local signature, both in space and frequency. Moreover, being a sounder, WHISPER does reveal the frequency position of $F_{\mathrm{lh}}$ even when no natural signatures are present. The difficulty, in the specific case of WHISPER measurements of $F_{\mathrm{lh}}$ frequency, is that no other resonances are accessible within the range of the instrument when this happens, and that neither reliable ion composition nor density derived from other instruments are available on board CLUSTER. Consequently, the challenge we had to face was to derive as much information as possible from a single measured parameter.

The $F_{\mathrm{lh}}$ value depends on three parameters: magnetic field amplitude, electron density and effective mass, which vary with plasma regime encountered (see Eq. 2, Sect. 3.1). The magnetic field amplitude can be estimated from a model, or measured with a good accuracy. The electron density and effective mass (related to composition in terms of the fractional abundance of ions present in the medium) are dependent on the geophysical situation encountered, while empirical, statistical, or theoretical models, are being elaborated (Craven et al., 1997; Gallagher et al., 2000; Pierrard and Lemaire, 2001). Actual measurements of the $F_{\mathrm{lh}}$ variable in the regions explored by CLUSTER thus form a valuable data set, bringing complementary information to current models. It is our ambition to construct a database of $F_{\text {lh }}$ measurement data, knowing that CLUSTER perigee is still decreasing. In this paper, we present observations from two recent case events, dated 12 July 2010 and 4 August 2010, chosen under conditions of low and moderate magnetic activity, respectively, in order to demonstrate the value of such data. In particular, density estimates (covering the range $2.10^{3}$ to $10.10^{3}$ electrons $\mathrm{cm}^{-3}$, at geocentric distances from 1.2 to $\left.3.4 R_{\mathrm{E}}\right)$ are shown for the two events, and discussed in the frame of the compared four spacecraft evolution along their respective orbits, and of the compared geomagnetic conditions.

The paper is organised as follows: Sect. 2 presents the context with more details, touching successively on WHISPER performance and evolution of the plasma regime encountered near perigee, Sects. 3 and 4 describe, respectively, data analysis and results obtained for the two events chosen, and Sect. 5 concludes with a summary of our results.

\section{Density estimation from the WHISPER instrument in the plasmasphere}

\subsection{Instrument performances}

The Whisper instrument, part of the WEC (Wave Experiment Consortium, Pedersen et al., 1997), combines two experiments to: (i) measure the natural emissions in the frequency band $2-80 \mathrm{kHz}$, and (ii) determine the electron density via a relaxation sounder (Trotignon et al., 2001). In both cases, the electric signal measured on one double sphere antenna by the EFW sensors (Gustafsson et al., 1997) is digitised and anal- ysed in frequency in the $2-80 \mathrm{kHz}$ bandwidth via an onboard FFT processor, delivering frequency spectra (power spectral density, amplitude only) at a resolution of 160 or $320 \mathrm{~Hz}$. Interface with telecommand and telemetry, as well as onboard data compression, is ensured by the DWP instrument (Woolliscroft et al., 1997).

In its sounder mode, the Whisper instrument operates as follows: the signal acquisition is performed a few milliseconds after the transmission of the sounder "pulse", a sine wave train of short duration ( 1.0 or $0.5 \mathrm{~ms}$ ). This pulse is centred at a frequency which sweeps the $4-80 \mathrm{kHz}$ bandwidth or a subpart of this) in successive frequency steps. Emitted sine pulses eventually trigger natural resonances in the medium; the resultant signal, amplified and acquired a few milliseconds after every step of the sounding sweep, is analysed in frequency, with a resolution of $160 \mathrm{~Hz}$. An "active" spectrum is built on board from a combination of relevant frequency slices associated with each step of the frequency sweep, and transmitted to the ground. A ground analysis of the resonance pattern present in the active spectrum allows identification of the plasma frequency $F_{\mathrm{p}}$ (Trotignon et al., 2001, 2010; Décréau et al., 2001). The sounder mode described above is operated alternatively with the natural wave mode (reception and analysis of waves naturally present in the medium). During natural mode operations, onboard frequency analysis is performed at a frequency resolution of either $160 \mathrm{~Hz}$ or $320 \mathrm{~Hz}$. Spectra returned to the ground always cover the $2-80 \mathrm{kHz}$ range. All four spacecraft follow the same time line, according to onboard time tagged operations, at a common precision of a one second boundary clock. Typically, $3 \mathrm{~s}$ of sounding mode (two sweeps) are followed by $49 \mathrm{~s}$ of natural wave mode (delivering 11 frequency spectra under nominal telemetry conditions). Satellite $\mathrm{C} 4$ capabilities in commanding sounding operations has been affected (on 25 September 2003) by a corruption of two bytes in the EEPROM where the onboard software is located. Since 13 December 2003, the C4 sounder works under a back up operation mode, with a sounding recurrence of $104 \mathrm{~s}$ where a single sounding sweep explores the $80-4 \mathrm{kHz}$ band in $1.1 \mathrm{~s}$. Onboard processing constraints lead to a time sequencing where $5 \mathrm{~s}$ of sounding mode operations are followed by $99 \mathrm{~s}$ of natural mode operations. As with satellites $\mathrm{C} 1, \mathrm{C} 2$ and $\mathrm{C} 3$, the frequency resolution of the returned active spectrum is $160 \mathrm{~Hz}$.

In the plasmasphere, which is the region of interest in this work, resonances arise at the electron cyclotron frequency $F_{\mathrm{ce}}$, its harmonics $n F_{\mathrm{ce}}$, the electron plasma frequency $F_{\mathrm{p}}$, the upper-hybrid frequency $F_{\mathrm{uh}}\left(F_{\mathrm{uh}}=\left(F_{\mathrm{p}}^{2}+F_{\mathrm{ce}}^{2}\right)^{1 / 2}\right)$, and $F_{\mathrm{qn}}$ which corresponds to Bernstein electrostatic cyclotron waves with group velocity close to zero $\left(F_{\mathrm{qn}}>2 F_{\mathrm{ce}}\right.$ and $\left.n F_{\mathrm{ce}}<F_{\mathrm{qn}}<(n+1) F_{\mathrm{ce}}\right)$. A detailed plasma diagnosis can therefore be made from the identification of these resonances, which depend on the magnetic field strength and the total electron density. Two populations of electrons, cold and hot, may also be revealed (Trotignon et al., 2003). 


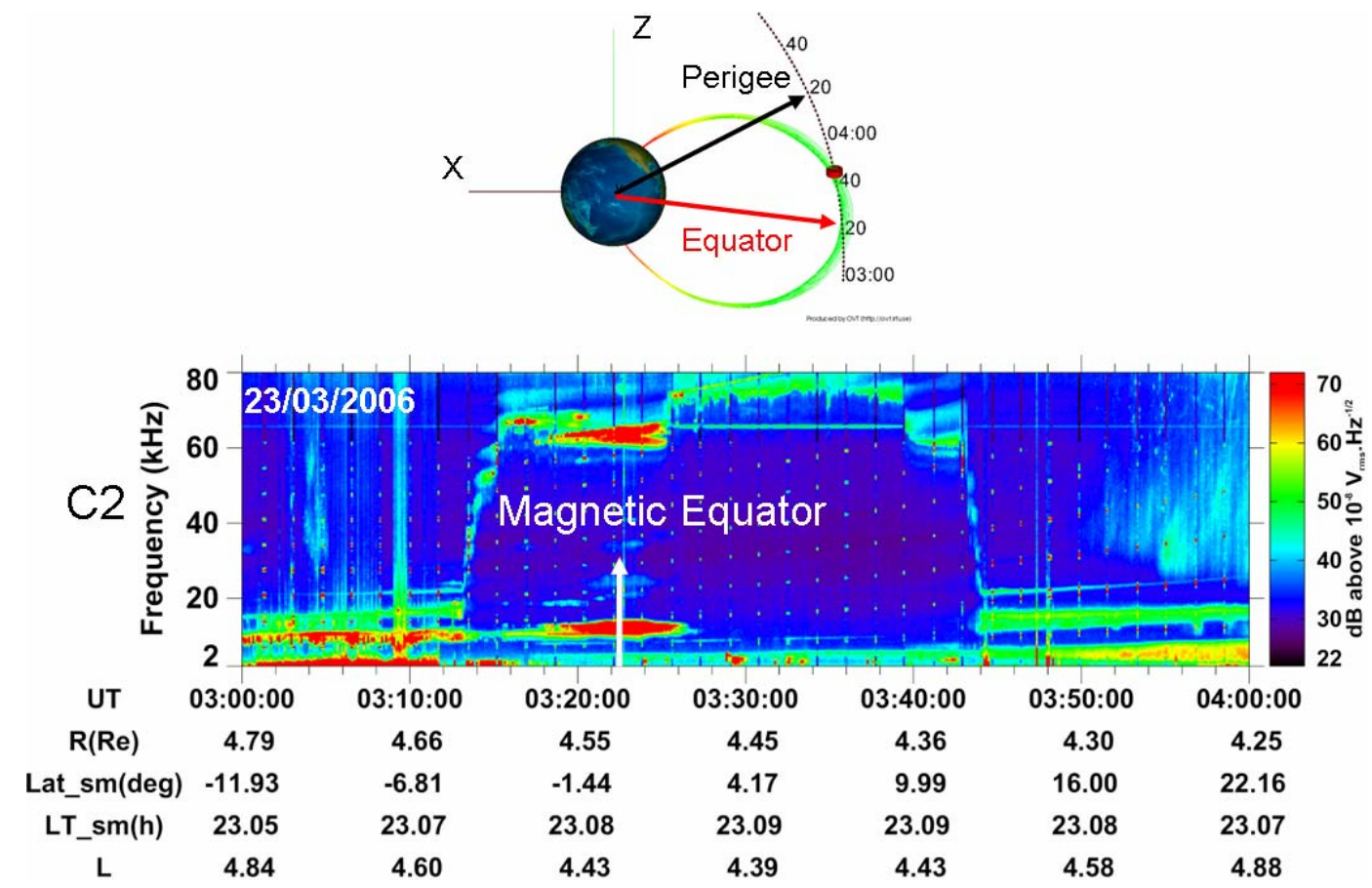

Fig. 1. Plasmasphere crossing during intermediate CLUSTER mission phase. Bottom panel shows the spectrogram recorded by C2 during this interval. Top panel is a GSM view of the C2 satellite trajectory, from minus Y, produced by the OVT tool (http://ovt.irfu.se). Both panels point out the magnetic equator, encountered inbound, about one hour before perigee. The $\mathrm{C} 2$ satellite grazes a plasmasphere boundary layer at $L \sim 4.5$, near midnight local time.

The most intense spectral energy density in the active spectrum is generally close to frequency $F_{\text {uh }}$, from which the plasma frequency (and thus the electron density) is currently derived. It has been indeed established from many observations in the magnetosphere (on ISEE-1, GEOS 1 \& 2 , Viking) that the resonances, including the $n F_{\text {ce }}$ series, are more intense close, and above, the plasma frequency and that the spectral energy density reaches its maximum very close to $F_{\text {uh }}$ (Trotignon et al., 1986). The magnetic field strength, and hence $F_{\mathrm{ce}}$, are known either from magnetic field measurements made by the FGM instrument (Balogh et al., 1997) or from $n F_{\text {ce }}$ observed by WHISPER $\left(F_{\mathrm{ce}}=28 B_{0}\right.$, where $F_{\mathrm{ce}}$ is expressed in $\mathrm{Hz}$ and $B_{0}$ in $\mathrm{nT}$ ).

It should be noted that, starting from a given $F_{\text {uh }}$ frequency value, measured at $\pm 160 \mathrm{~Hz}$ uncertainty, the precision attached to a derived density depends sensibly upon the value of the ratio $F_{\mathrm{p}} / F_{\mathrm{ce}}$, that is upon the plasma regime encountered. Precision can be poor when $F_{\mathrm{p}} / F_{\mathrm{ce}} \ll 1$. An example of weakly magnetized plasma is shown in Fig. 1, near 03:15 UT $\left(F_{\mathrm{p}} / F_{\mathrm{ce}} \sim 8\right.$, with $F_{\mathrm{p}}=55.6 \mathrm{kHz}$ and $F_{\mathrm{ce}}=$ $6.9 \mathrm{kHz})$, and a more strongly magnetized plasma $\left(F_{\mathrm{p}} / F_{\mathrm{ce}} \sim\right.$ 0.35 , with $F_{\mathrm{p}}=22.9 \mathrm{kHz}$ and $\left.F_{\mathrm{ce}}=67.7 \mathrm{kHz}\right)$ is encountered near 15:35 UT in the spectrogram displayed in Fig. 2. With similar values of the measured $F_{\mathrm{uh}}$ quantity, the precision obtained for derived density values is, in the first case, roughly one order of magnitude better than in the second case (where $\left.\Delta N_{\mathrm{e}} / N_{\mathrm{e}} \sim 5 \%\right)$.

\subsection{Plasma conditions near CLUSTER perigee}

The plasmasphere region was not a primary scientific objective of CLUSTER, and the WHISPER frequency range was not perfectly tuned to measure plasma frequency profiles along each plasmasphere crossing at perigee. Moreover, particle analyzer measurements are difficult in this region, by the nature of the plasma regime encountered (presence of a photoelectron sheath surrounding spacecraft), and also due to the proximity of the radiation belts, bearing dangerous environmental conditions. The electron PEACE instrument (Johnstone et al., 1997) has been thus often switched off near perigee. However, the plasmaphere, governed by large scale electric fields, is responding in a complex manner to geomagnetic activity. The whole region, coupled to the radiations belts and current rings, hosts a large number of waves, over a large range of frequencies. Physical processes in the plasmasphere are, still now, not fully understood, and the object of utmost attention (Darrouzet et al., 2009a), noting in particular their importance in the space weather context.

During the first years of the CLUSTER mission, the CLUSTER orbit crossed the plasmasphere region at almost every perigee pass, exceptions being encountered under very high magnetic activity conditions, when the plasmasphere is eroded down to low geocentric distances. Perigee is placed not far from the magnetic equator, and it happens that the orbit grazes the plasmasphere boundary layer along 
a)

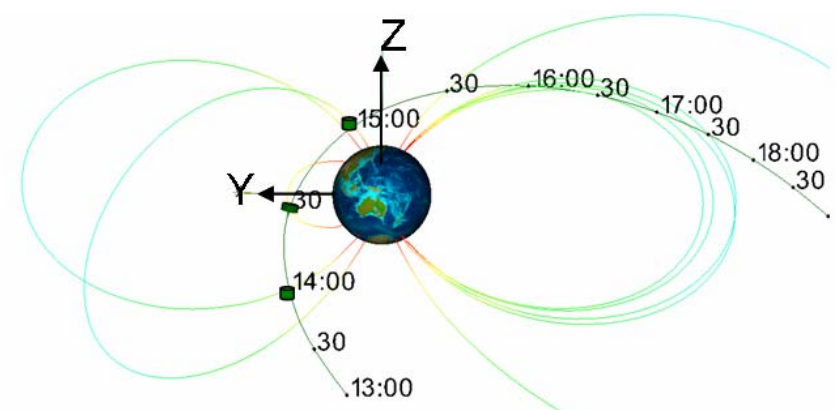

b)

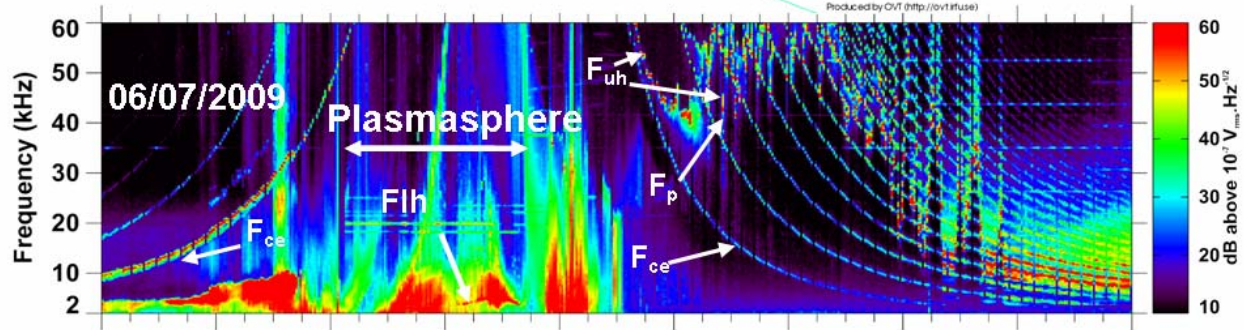

$\begin{array}{ccccccccccc}\text { UT } & 12: 30: 00 & 13: 10: 00 & 13: 50: 00 & 14: 30: 00 & 15: 10: 00 & 15: 50: 00 & 16: 30: 00 & 17: 10: 00 & 17: 50: 00 & 18: 30: 00 \\ \text { R(Re) } & 5.84 & 4.57 & 3.17 & 1.88 & 2.12 & 3.50 & 4.87 & 6.12 & 7.24 & 8.28 \\ \text { Lat_sm(deg) } & -70.46 & -71.93 & -58.10 & -7.48 & 75.83 & 47.96 & 29.44 & 17.87 & 9.50 & 2.92 \\ \text { LT_sm(h) } & 0.63 & 22.08 & 19.26 & 17.67 & 12.40 & 6.73 & 6.31 & 6.20 & 6.17 & 6.16 \\ \text { L } & 53.86 & 52.37 & 11.56 & 1.91 & 26.98 & 7.64 & 6.23 & 6.52 & 7.15 & 7.90\end{array}$

c)

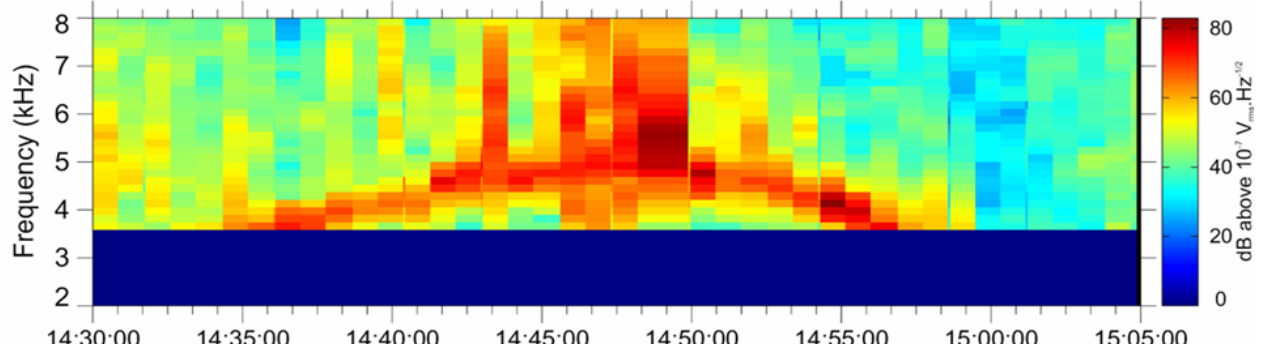

UT
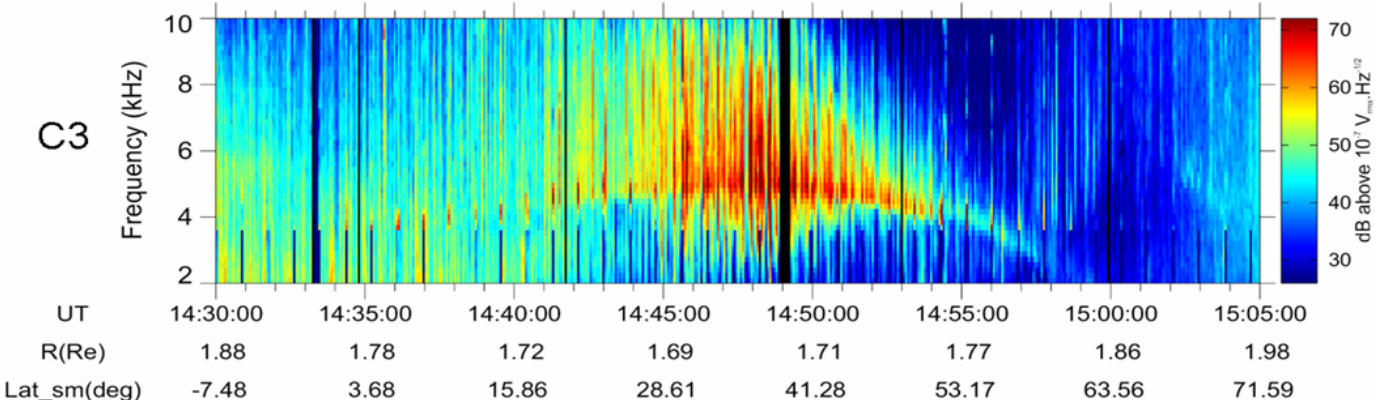

14:40:00

14:50:00

14:55:00

15:00:00

:05:00

Lat_sm(deg)

$-7.48$

3.68

15.86

28.61

41.28

53.17

63.56

71.59

L

1.91

1.78

17.27

17.03

16.72

16.27

15.58

14.38

1.84

2.15

2.88

4.45

7.93

15.50

Fig. 2. Crossing of the plasmasphere typical of the late mission phase, here on 6 July 2009. (a) orbit of the C3 satellite and field lines in a GSM coordinate system with view from $-X$, produced by the OVT tool. (b) WHISPER spectrogram of combined active and passive spectra. C3 crosses the plasmasphere successively in the dusk and dawn sectors, under different conditions of altitude and L values. (c) Zoom of $F_{\mathrm{lh}}$ observations near perigee. Top: spectrogram constructed from active spectra. Bottom: spectrogram combining active and passive observations.

a significant distance $\left(\sim 1 R_{\mathrm{E}}\right)$ below and above the equatorial plane, within a narrow local time sector (Décréau et al., 2005). More generally, the WHISPER frequency range, al- though excluding density values above $80 \mathrm{~cm}^{-3}$, describes adequately the outer plasmasphere region where plasma plumes and other irregularities are encountered (Darrouzet et 
al., 2006, 2009b). A systematic study of the 2002-2003 data (El-Lemdani Mazouz et al., 2009), gives a precise view of the circumstances in which WHISPER upper density threshold is exceeded. The study shows that this limit is reached in an average of about $45 \%$ of the perigee passes, with a higher occurrence in the afternoon sectors (up to $70 \%$ in the 16:00-18:00 LT interval), and a lower occurrence in the morning sectors (down to $22 \%$ in the 04:00-06:00 LT interval). This variation points out the dawn-dusk asymmetry of the plasmasphere due to the presence of higher densities near the dusk bulge. In regions beyond the WHISPER accessibility domain, and when densities are not too high, the measurements of spacecraft potential by the EFW electric field instrument lead to an estimate of density values (Moullard, 2002). Spacecraft potential values have to be associated with WHISPER measurements in order to enable calibrations of EFW potential measurements. Another interesting feature of the CLUSTER orbit is its high inclination angle. It allows in particular an estimate with a good precision of the latitudinal extension of electrostatic waves confined to regions near the magnetic equator (El-Lemdani Mazouz et al., 2009). Indeed, some orbit crossings of the plasmaphere body provide almost symmetrical views, with respect to the equatorial plane, over density and waves (Fig. 1 in Décréau et al., 2001, Figs. 7 and 9 in Darrouzet et al., 2009b).

Most often, the magnetic dipole axis is tilted with respect to the plane perpendicular to the ecliptic, and CLUSTER encounters higher magnetic field values outbound (in the Northern Hemisphere) than inbound (Fig. 4 in Rauch et al., 2006). As time goes by, the asymmetry between the inbound orbit element and the outbound orbit element increases, due to a displacement of apogee below the ecliptic plane, associated with a smaller orbit inclination. Later in the CLUSTER mission phase, the orbit undergoes important evolutions, namely a larger argument of periapsis (the apogee moves southwards) and a perigee descent.

Figure 1 illustrates a plasmasphere crossing in an intermediate situation, encountered in March 2006, with perigee still at high $\left(4.2 R_{\mathrm{E}}\right)$ geocentric distance, but with a significant argument of periapsis. In the case event shown, the CLUSTER C2 satellite, travelling inbound, encounters a minimum in magnetic field magnitude at 03:07 UT, before entering the plasmasphere. The satellite penetrates in this region, at $L \sim 4.5$, shortly before encountering the magnetic equator (pointed out by an arrow). It leaves the plasmasphere shortly afterwards, at a positive magnetic latitude $\left(13^{\circ}\right)$. The satellite travels still inbound after plasmasphere traversal, and the magnetic field is still increasing, as shown by the resonances at $F_{\mathrm{ce}}$ and harmonics present (green dots) in the WHISPER spectrograms. The perigee is reached at $\sim 04: 20$ UT, after the time interval covered by the spectrogram.

Figure 2 illustrates the typical situation encountered from 2008 onwards, here on 6 July 2009. A large portion of the orbit around perigee is shown in Fig. 2a. Perigee is reached near 14:45 $\mathrm{UT}$, at about $1.7 R_{\mathrm{E}}$ geocentric distance and magnetic latitude $\sim 30^{\circ}$. Wave signatures obtained by WHISPER are presented in the spectrogram shown in Fig. 2b. Near perigee, the satellite travels at low altitudes, where high values of magnetic field magnitude are associated with $F_{\text {ce }}$ frequency values going above the upper limit of the Whisper instrument frequency range. In the case event chosen, $F_{\mathrm{ce}}$ resonances and harmonics triggered by the sounder are showing up clearly, as lines equidistant in frequency, before 13:55 UT and after 15:35 UT. From 13:55 to 15:35 UT, $F_{\text {ce }}$ with $F_{\text {uh }}$ being above the instrument's working frequency range, WHISPER has no access to direct signatures of density values (via sounder resonances). The local gyrofrequency is measured by the onboard magnetometer FGM and can also be deduced from a model (e.g. Tsyganenko and Sitnov, 2005, used in this study). Plasma density, in contrast, is not measured on board (in those regions particle analyzers are either not fully operational, or do not have access to the cold population). However, indirect signatures of plasmapause crossings are provided by man made signals, ground transmitter lines at constant frequency which, created inside the plasmasphere, are reflected at the plasmapause and cannot escape. The line observed at $f_{0} \sim 20 \mathrm{kHz}$, for instance, is interrupted at the supposed plasmapause boundary encountered inbound at $\sim 13: 55 \mathrm{UT}, \sim 18.8 \mathrm{MLT}, L \sim 7.5$, and geocentric distance $R \sim 3 R_{\mathrm{E}}$. Outbound, the signature from $f_{0}$, not as clear, is seen at $\sim 15: 00 \mathrm{UT}, \sim 16.0 \mathrm{MLT}, L \sim 7.9$, $R \sim 1.9 R_{\mathrm{E}}$. From 15:00 to 15:35 UT, CLUSTER C3 travels over the polar cap. After 15:35 UT, the $F_{\text {uh }}$ resonance is clearly observed, as well as the $F_{\mathrm{p}}$ resonance when the $F_{\text {uh }} / F_{\text {ce }}$ ratio is above $1.4\left(F_{\mathrm{p}}>F_{\text {ce }}\right)$. In practice, when $F_{\mathrm{p}} / F_{\mathrm{ce}}>\sim 3$ (after 16:20 UT and before 17:45 UT) both resonances, close to each other, are seen as a single peak in the active spectra. The frequency positions of resonances allows us to follow a density profile inside the plasmasphere boundary, with Ne values, on average, increasing (with $F_{\text {uh }}$ ) from 15:35 to $\sim 16: 30 \mathrm{UT}$, then decreasing. A great number of density irregularities are observed along that orbit element, located inside the plasmapause boundary layer $(L \sim 6.2-$ 7.5 ), in the dawn sector.

Inside the dusk side inner plasmasphere, a rich variety of natural emissions are visible in the WHISPER frequency range that will be described in another paper. One category displays a lower frequency cut off which we identify as the local lower hybrid frequency $\left(F_{\mathrm{lh}}\right)$, identified by a white arrow. Figure $2 \mathrm{c}$ displays a zoom of the WHISPER spectrogram in the frequency-time domain of interest. The bottom panel is a spectrogram combining spectra obtained during natural mode operations (lower frequency at $2 \mathrm{kHz}$ ) and sounding mode operations (lower frequency $\sim 3.8 \mathrm{kHz}$ ); the upper panel displays only active spectra (pixels enlarged in time for better visibility). The triggered resonances are characterized by high spectral energy densities (red colour coded). Their frequencies are interpreted as being placed at $F_{\mathrm{lh}}$ because this characteristic frequency is the best (and 

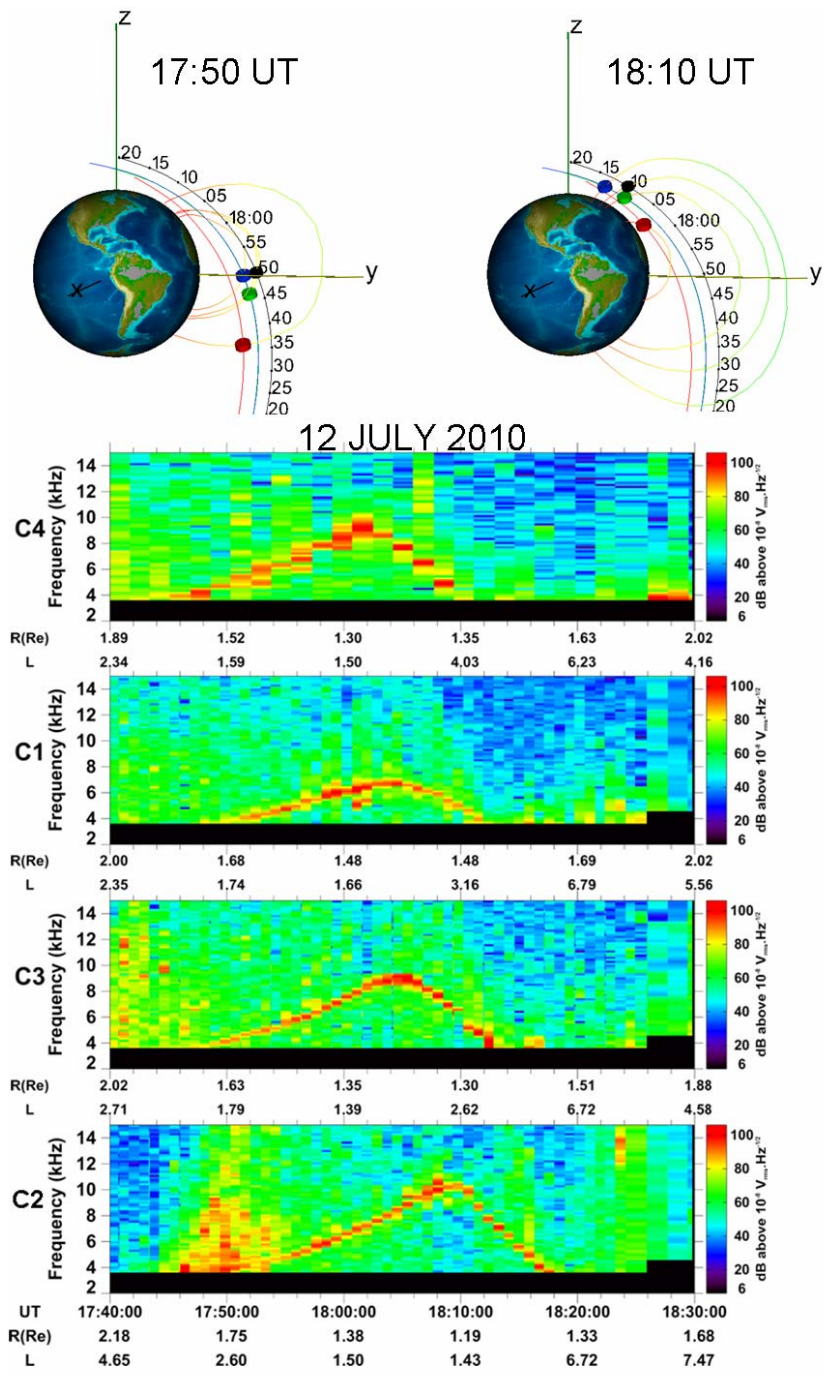

Fig. 3. 12 July 2010 event. Top: CLUSTER orbits in the SM coordinate system. Views produced by OVT tool. Bottom: WHISPER spectrograms focused on lower hybrid resonances (active spectra only).

only) candidate in this frequency range under the plasma conditions encountered. Observed resonances are actually close to the local $F_{\text {ce }}(225 \mathrm{kHz}$ at $14: 44 \mathrm{UT})$ multiplied by $\sqrt{m_{\mathrm{e}} / m_{\mathrm{p}}}$, i.e. the $F_{\mathrm{lh}}$ value expected in a proton plasma of high density $\left(F_{\mathrm{p}} / F_{\mathrm{ce}}>1\right)$. Moreover, those resonances are placed at the low frequency limit of a wedge like signature present in the spectrogram shown in the lower panel of Fig. 2c. This spectrogram combines active and passive spectra, and the dimensions of the pixels along the time coordinate are about one tenth the size of the pixels in the above spectrum in order to fit in all of the measurements. Wedge like spectrograms have been observed by the DEMETER satellite (Shklyar et al., 2010), and their lower frequency cut off has been interpreted as the local $F_{\mathrm{lh}}$.

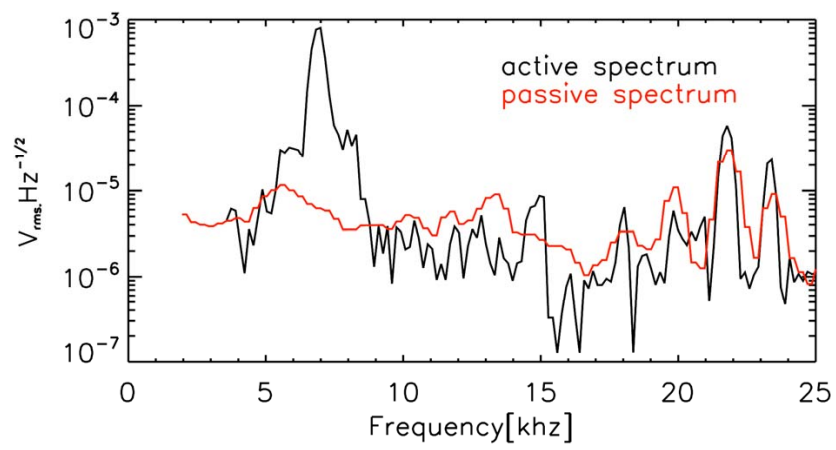

Fig. 4. Detailed view of the $F_{\mathrm{lh}}$ resonance triggered by the WHISPER sounder on C2 satellite, at 18:04 UT, on 12 July 2010. The active spectrum (black line) is compared to the passive spectrum measured $2 \mathrm{~s}$ later (red line).

The event shown in Fig. 2 is one of the first examples of that kind noticed in the CLUSTER/WHISPER data. Since then, similar events have been observed almost systematically near perigee, more and more clearly as the perigee altitude decreases. Figure 3 presents a case event taken about a year after the one shown in Fig. 2. Perigee altitudes have decreased from $\sim 4500 \mathrm{~km}$ down to $\sim 1200 \mathrm{~km}$. The views on top of Fig. 3 present the four orbits projected on the Y$\mathrm{Z}$ plane in the GSM coordinate system, C3 following C4 on the same orbit. Spacecraft positions and associated magnetic field lines are displayed at two times, respectively 17:50 UT and 18:10 UT. The panels at the bottom of Fig. 3 display the four WHISPER spectrograms constructed from active spectra between 17:40 and 18:30 UT. They point out clearly the presence of a single resonance, generally of good quality, well isolated in the active spectrum, in the 17:45-18:15 UT time frame. This is illustrated in Fig. 4, which displays the active frequency spectrum derived at 18:04 UT on C2, compared to the passive frequency spectrum derived two seconds later. The triggered $F_{\mathrm{lh}}$ resonance peaks about two of magnitude above natural emissions. For both case events, 6 July 2009 and 12 July 2010, the spacecraft are located in the dusk sector, and $F_{\mathrm{lh}}$ resonances are seen briefly near perigee, at small values of the Mac Ilwain parameter $L$. The upper value of the lower hybrid frequency has increased from $\sim 5 \mathrm{kHz}$ (earlier event) to $\sim 10 \mathrm{kHz}$ (most recent event).

We shall leave to future studies the fine analysis of resonance characteristics, in particular how the signal magnitude is related to antenna orientation with respect to the magnetic field, and how it behaves with respect to the level of transmission. As an indication, the electric field intensity at resonances is two orders of magnitude higher than that of natural emissions present in the medium, the level of transmitted sine wave train being high ( $200 \mathrm{~V}$ peak to peak). For a general description of electron resonances in space (case of topside sounders) we refer to the review by Muldrew (1972) and, for the specific case of the relaxation sounder behaviour, to 
Etcheto and Bloch (1978). If the frequency of the transmitted sine wave train is close to a resonance frequency of the medium (low group velocity), the medium exhibits a sharp frequency response. This phenomenon is interpreted either as waves moving along the satellite (group velocity equal to satellite velocity relative to the plasma) or as oblique echoes. The latter view is interpreted as plasma anisotropy modifying the group path for waves close to the resonance frequency, so that the path is bent over and forms a closed loop, returning the transmitted wave packet to the sounder's receiver. For a theoretical description of the medium properties near the $F_{\mathrm{lh}}$ resonance, we refer to Béghin and Debrie (1972). In this paper, the authors describe observations, from an ejected nose-cone payload, of waves created in the vicinity of $F_{\mathrm{lh}}$ by a transmitting antenna placed on a rocket payload. They present the dispersion diagram in the perpendicular direction, in the frame of kinetic theory, for the generalized ion Bernstein modes above $F_{\mathrm{lh}}$. Dispersion solutions, found in each harmonic band of the ion gyro-frequency, are compared to an adiabatic dispersion equation similar to Bohm and Gross's equation obtained in the frame of hydrodynamic theory for electron plasma waves. Béghin and Debrie (1972) show that the adiabatic solution is the locus of the zero group velocity points of the kinetic theory (see Fig. 3 of their paper). Although they refer to ionospheric plasma (oxygen as main constituent), in contrast with plasma at CLUSTER's altitudes (hydrogen as main constituent), we think that the dispersion diagram to be expected in the situation encountered by CLUSTER is formally similar. Plasma behaves globally, in the vicinity of $F_{\mathrm{lh}} \gg F_{\mathrm{i}}\left(F_{i}\right.$ refering here to the $\mathrm{H}^{+}$ion gyrofrequency), according to a single branch of the dispersion diagram, formally identical to hydrodynamic theory for electron plasma waves in the vicinity of the plasma frequency (case of unmagnetized plasma, $F_{\mathrm{p}} \gg F_{\mathrm{ce}}$ ). In both cases, the dispersion relation is written as:

$\omega^{2}=\omega_{0}^{2}+v_{\mathrm{T}}^{2} k^{2}$

where $\omega$ and $k$ are pulsation and wave number variables, $\omega_{0}$ is the pulsation related to the plasma frequency (respectively the lower hybrid frequency) and $v_{\mathrm{T}}$ is the thermal electron velocity (respectively a hybrid thermal velocity). In both cases, the relaxation sounder creates a single resonance at pulsation $\omega_{0}$ which is a solution of the dispersion relation (1) at zero group velocity. The next section presents plasma diagnostics performed for the case event shown in Fig. 3, assuming that resonances are placed precisely at frequency $F_{\mathrm{lh}}$.

\section{Derivation of plasma frequency from $F_{\text {lh }}$ measurement}

\subsection{General problem}

The $F_{\mathrm{lh}}$ value responds to equation (e.g. see Masson et al., 2009):

$1 /\left[M_{\text {eff }}\left(m_{\mathrm{p}} / m_{\mathrm{e}} \cdot F_{\mathrm{lh}}^{2}\right)\right]=1 / F_{\mathrm{p}}^{2}+1 / F_{\mathrm{ce}}^{2}$,

where $m_{\mathrm{p}}$ and $m_{\mathrm{e}}$ are, respectively, the proton and electron masses and $M_{\text {eff }}$ the effective ion mass, a dimensionless factor depending on the fractional abundance of ions present in the medium. When considering the three major constituents $M_{\text {eff }}$ is defined as:

$1 / M_{\mathrm{eff}}=\alpha / 1+\beta / 4+\gamma / 16$,

where $\alpha, \beta$ and $\gamma$ are respectively the fractional abundances of $\mathrm{H}^{+}, \mathrm{He}^{+}$and $\mathrm{O}^{+}$. The region of interest for the chosen case event is the inner plasmasphere at low altitude. The domains of the Mc Ilwain parameter $L$ and the geocentric distance $R$ which are swept by the different satellites are roughly $1<L<4$ and $1.2 R_{\mathrm{E}}<R<1.8 R_{\mathrm{E}}$, respectively, hence in a zone above the ionosphere and well inside closed magnetic field lines. There, $F_{\mathrm{ce}}$ values deduced from a magnetic field model are very close to actual values. The magnitude of the magnetic field is measured by the onboard magnetometer instrument, FGM, in this case in the upper part of its dynamical range. A comparison between models and onboard measurements shows an excellent agreement, better than $1 \%$ for all spacecraft during time intervals of interest. In that case magnetic field models can be reduced to the contribution of the IGRF model (Macmillan et al., 2003), but full magnetospheric models (Tsyganenko and Sitnov, 2005) have been tested.

Equation (2) leads to simple solutions in cases where the ratio $F_{\mathrm{p}} / F_{\mathrm{ce}}$ is either much larger or much smaller than 1 . In the first situation, $F_{\mathrm{lh}}$ takes the limit value:

$F_{\mathrm{lh}}=F_{\mathrm{lh} 0} / \sqrt{M_{\mathrm{eff}}}$,

where

$F_{\mathrm{lh} 0}=F_{\mathrm{ce}} \sqrt{m_{\mathrm{e}} / m_{\mathrm{p}}}$

is a quantity governed solely by $F_{\text {ce }}$, which is known with a good accuracy. Measurement of $F_{\mathrm{lh}}$ in that context provides a direct estimate of the effective ion mass $M_{\text {eff. }}$. It is interesting to note that, $M_{\text {eff }}$ being larger than or equal to $1, F_{\mathrm{lh}}$ is smaller than $F_{\mathrm{lh} 0}$. Let's define the value of $M_{\text {eff } \_}=\left(F_{\mathrm{lh} 0} / F_{\mathrm{lh}}\right)^{2}$, the asymptotic value of the quantity $\left(F_{\mathrm{lh} 0} / F_{\mathrm{lh}}\right)^{2}$, for $F_{\mathrm{p}}$ close to infinity. If $F_{\mathrm{p}}$ decreases $\left(M_{\mathrm{eff}}\right.$ and $F_{\text {ce }}$ being stable), Eq. (2) indicates that $\left(F_{\mathrm{lh} 0} / F_{\mathrm{lh}}\right)^{2}$ increases above the actual value of $M_{\text {eff }}, M_{\text {eff } \_0}$. Hence, $\left(F_{\mathrm{lh} 0} / F_{\mathrm{lh}}\right)^{2}$ being always equal to or higher than $M_{\text {eff }}$, we can use the former quantity as the highest threshold for the value $M_{\text {eff }}$ under estimation, and introduce:

$M_{\text {eff_high }}=\left(F_{\mathrm{lh} 0} / F_{\mathrm{lh}}\right)^{2}$. 
When $F_{\text {ce }}$ is much larger than $F_{\mathrm{p}}$, Eq. (2) leads to:

$$
F_{\mathrm{p}}=F_{l h} \cdot \sqrt{M_{\mathrm{eff}}\left(m_{\mathrm{p}} / m_{\mathrm{e}}\right)} .
$$

The plasma frequency and lower hybrid frequency are proportional to each other. Measurement of $F_{\mathrm{lh}}$ in that context provides a direct estimate of the plasma frequency $F_{\mathrm{p}}$, should the effective mass $M_{\text {eff }}$ be known independently.

Figure 5d shows how asymptotic limits are reached, by plotting variations of $F_{\mathrm{lh}} / F_{\mathrm{lh} 0}$ as a function of $F_{\mathrm{ce}} / F_{\mathrm{p}}$, for the particular case of $M_{\mathrm{eff}}=1\left(\mathrm{H}^{+}\right.$plasma $)$. In practice, limit cases are reached respectively when $F_{\mathrm{ce}} / F_{\mathrm{p}}<0.25$ ( $F_{\mathrm{lh}}$ can then be used as a measure of $\left.M_{\text {eff }}\right)$ and when $F_{\mathrm{ce}} / F_{\mathrm{p}}>4\left(F_{\mathrm{lh}}\right.$ is then proportional to $F_{\mathrm{p}}$ ). The CLUSTER orbit offers fortuitously a unique and interesting feature during observations of the $F_{\mathrm{lh}}$ resonance: $F_{\mathrm{ce}} / F_{\mathrm{p}}$ ratio increases along the orbit from values lower than unity to values above unity. One can thus take advantage, in turn, of the two different facets offered by $F_{\mathrm{lh}}$ measurements, and evaluate the two different plasma parameters they are revealing.

\subsection{Specific situation encountered on CLUSTER}

Figure 5 presents the analysis and interpretation of the $F_{\text {lh }}$ frequency values measured along the orbit of the CLUSTER C2 satellite during the case event of 12 July 2010. The upper panel (Fig. 5a) completes the orbit views shown in Fig. 3. This projection onto a plane centred on Earth, from a view point not far from the Y-axis, displays magnetic field lines crossed by $\mathrm{C} 2$ at 5 min intervals from 17:45 to 18:20 UT; the magnitude of the magnetic field along the field lines are colour-coded (increasing values from green to red). The smallest $\mathrm{L}$ value $(L=1.23)$ is reached at $\sim 18: 05 \mathrm{UT}$, followed by perigee (altitude $1210 \mathrm{~km}$ ) at $\sim 18: 11 \mathrm{UT}$ and the highest magnetic field intensity $(25900 \mathrm{nT})$ at $\sim 18: 16 \mathrm{UT}$. This view emphasizes the fact that, during the limited time interval $(\sim 20 \mathrm{~min})$ when $F_{\mathrm{lh}}$ estimates are available, the satellite, moving at about $9 \mathrm{~km} \mathrm{~s}^{-1}$, explores significant intervals in space (precise altitude, $L$ and MLT variations versus time are plotted in Fig. 7a).

Figure $5 \mathrm{~b}$ shows the variation with time of the measured $F_{\mathrm{lh}}$ frequency (red solid line) when available, i.e. for $F_{\mathrm{lh}} \geq$ $4 \mathrm{khz}$. The two red dashed lines, hardly visible because they partially overlap the solid red curve, are neighbouring frequencies, $F_{\mathrm{lh}} \pm \Delta f$, where $\Delta f=160 \mathrm{~Hz}$ is the accuracy of the WHISPER frequency measurements. $F_{\mathrm{lh}}$ values are compared to $F_{\mathrm{lh} 0}$ (black line) given by Eq. (4), using an $F_{\mathrm{ce}}$ variation from the modelled magnetic field. $F_{\mathrm{lh} 0}$ is actually directly proportional to $F_{\mathrm{ce}}$. At the beginning of the time interval, both frequencies differ by a small percentage as the red curve can be derived from the black one by a translation along the ordinate of a small amount, indicating: (i) a value of $M_{\text {eff }}$ close to $1\left(F_{\mathrm{lh} 0} \sim F_{\mathrm{lh}}\right)$, (ii) a plasma regime where Eq. (3) ( $F_{\mathrm{lh}}$ proportional to $\left.F_{\mathrm{lh} 0}\right)$ is valid, thus $F_{\mathrm{ce}} \ll F_{\mathrm{p}}$. Under this condition, $M_{\text {eff }}$ can be directly calculated from
Eq. (3). As time increases, $F_{\mathrm{lh}}$ measured frequencies (red curve) culminate, then decrease, while $F_{\mathrm{lh} 0}$ values continue to rise (black curve). Actually, the ratio $F_{\mathrm{lh}} / F_{\mathrm{lh} 0}$ decreases from a constant value $\sim 0.9$, between 17:53 and 18:05 UT, to $\sim 0.8$ at 18:08 UT, 0.55 at 18:11, and finally down to 0.22 at 18:16 UT. This behaviour mimics the $F_{\mathrm{lh}} / F_{\mathrm{lh} 0}$ variations shown in Fig. 5d, indicating that $F_{\text {ce }} / F_{\mathrm{p}}$ increases continuously with time, and $F_{\mathrm{ce}} \gg F_{\mathrm{p}}$ at the end of the observations. The green line shows $F_{\mathrm{lh}}$ variations versus time according to Eq. (3), thus still proportional to $F_{\text {ce }}$, where $M_{\text {eff }}$ is deduced from a best fit between experiment and Eq. (3) at the beginning of the time interval.

Figure $5 \mathrm{c}$ displays variations of the $F_{\mathrm{p}}$ values (noted fpe) calculated from Eq. (2) under two assumptions for the $M_{\text {eff }}$ value, respectively $M_{\text {eff }}=1$ (black solid line) and $M_{\text {eff }}=$ 1.26 (green solid line), i.e. the value deduced from the best fit shown in Fig. 5b. Variations of the electron gyro-frequency (and the same value multiplied by 3 ) are plotted as dotted black lines. This panel illustrates the benefits and drawbacks of the various situations (sorted by order of magnitude of the ratios $F_{\mathrm{ce}} / F_{\mathrm{p}}$ ) encountered respectively at the beginning, the medium part, and the end of the time interval covered by the $F_{\mathrm{lh}}$ measurements. We will now discuss which kind of diagnostic can be derived along the three following successive time intervals: (i) $17: 53$ to $18: 05 \mathrm{UT}\left(F_{\mathrm{lh}} / F_{\mathrm{lh} 0} \sim 0.9\right)$; (ii) $18: 05$ to $18: 13 \mathrm{UT}\left(0.5<F_{\mathrm{lh}} / F_{\mathrm{lh} 0}<0.9\right)$ and $18: 13$ to 18:17 UT $\left(F_{\mathrm{lh}} / F_{\mathrm{lh} 0}<0.5\right)$, marked I, II, and III in Fig. 5.

The first interval is devoted to the estimation of the effective mass $M_{\text {eff }}$, as described above. In practice, the best fit of Eq. (3) with experiment (green plot in Fig. 5b), is placed inside the interval corresponding to the frequency uncertainty interval $( \pm 160 \mathrm{~Hz})$. The relative uncertainty of the $M_{\mathrm{eff}}$ estimate can be evaluated as $\pm 5 \%\left(\sim 2 \Delta F_{\mathrm{lh}} / F_{\mathrm{lh}}\right)$, which is also roughly the level of fluctuations of that quantity over the time interval examined. This leads to an $M_{\text {eff }}$ value that is nearly stable (between 1.2 and 1.3) which is valid here near MLT $=18$ for a rather large interval in L values and altitudes, both decreasing along the orbit from 2.19 to 1.26 and from $\sim 4000$ to $1600 \mathrm{~km}$, respectively. During this first time sequence, no $F_{\mathrm{p}}$ value can be derived safely from Eq. (2), as the $F_{\mathrm{lh}}$ value does not depend significantly on $F_{\mathrm{p}}$. However, $F_{\mathrm{p}}$ variations based on an $M_{\mathrm{eff}}=1$ assumption (black curve peaking near 18:06 UT) provides a lower $F_{\mathrm{p}}$ threshold since any other physical value of $M_{\text {eff }}(>1)$ would yield higher values, as illustrated by the few points of the green plot derived during that interval.

During the second selected time interval, from 18:05 to 18:13 UT, we treat $F_{\mathrm{lh}}$ measurements by assuming that the ion composition is the same as that encountered during the first interval. The calculated plasma-frequency profile is then the green plot shown in Fig. 5c. This approach yields a first approximation, which is qualitatively useful, although ion composition is likely to vary during this second time interval. Actually, the diffusive equilibrium model (Angerami and Thomas, 1964; see also Sonwalkar et al., 2011b) indicates 


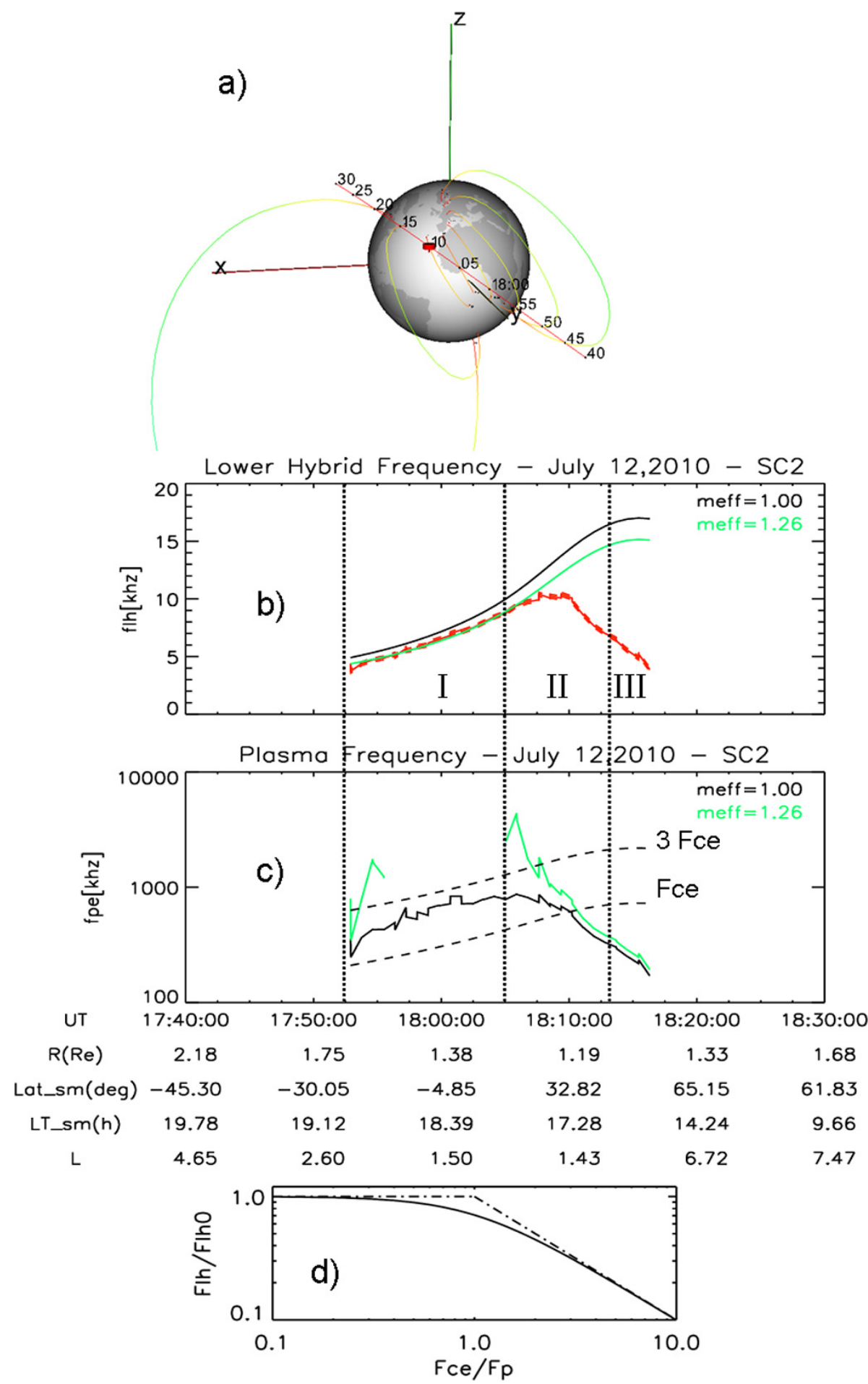

Fig. 5. Variation of magnetic field intensity and plasma frequency on C2, event of 12 July 2010. (a) Magnetic field lines crossed by C2 from 17:45 to 18:20 UT, where the magnetic field intensity is colour coded (decreasing values from red to green). View produced by OVT tool. (b) $F_{\mathrm{lh}}$ variations in the limiting case of infinite $F_{\mathrm{p}}\left(F_{\mathrm{lh}}\right.$ proportionnal to $\left.F_{\mathrm{ce}}\right)$ under two assumptions about effective mass, either $M_{\mathrm{eff}}=1$ as given by the black (upper-most) curve or $M_{\mathrm{eff}}=1.26$ as given by the green curve. Measured $F_{\mathrm{lh}}$ values are plotted in red. Different relative variations are pointed out by splitting time into intervals I, II, and III; (c) derived plasma frequency for the two ion compositions tested. (d) theoretical variations (with $M_{\mathrm{eff}}=1$ ) showing which of $F_{\mathrm{p}}$ or $F_{\mathrm{ce}}$ parameter governs the value $F_{\mathrm{lh}}$. 

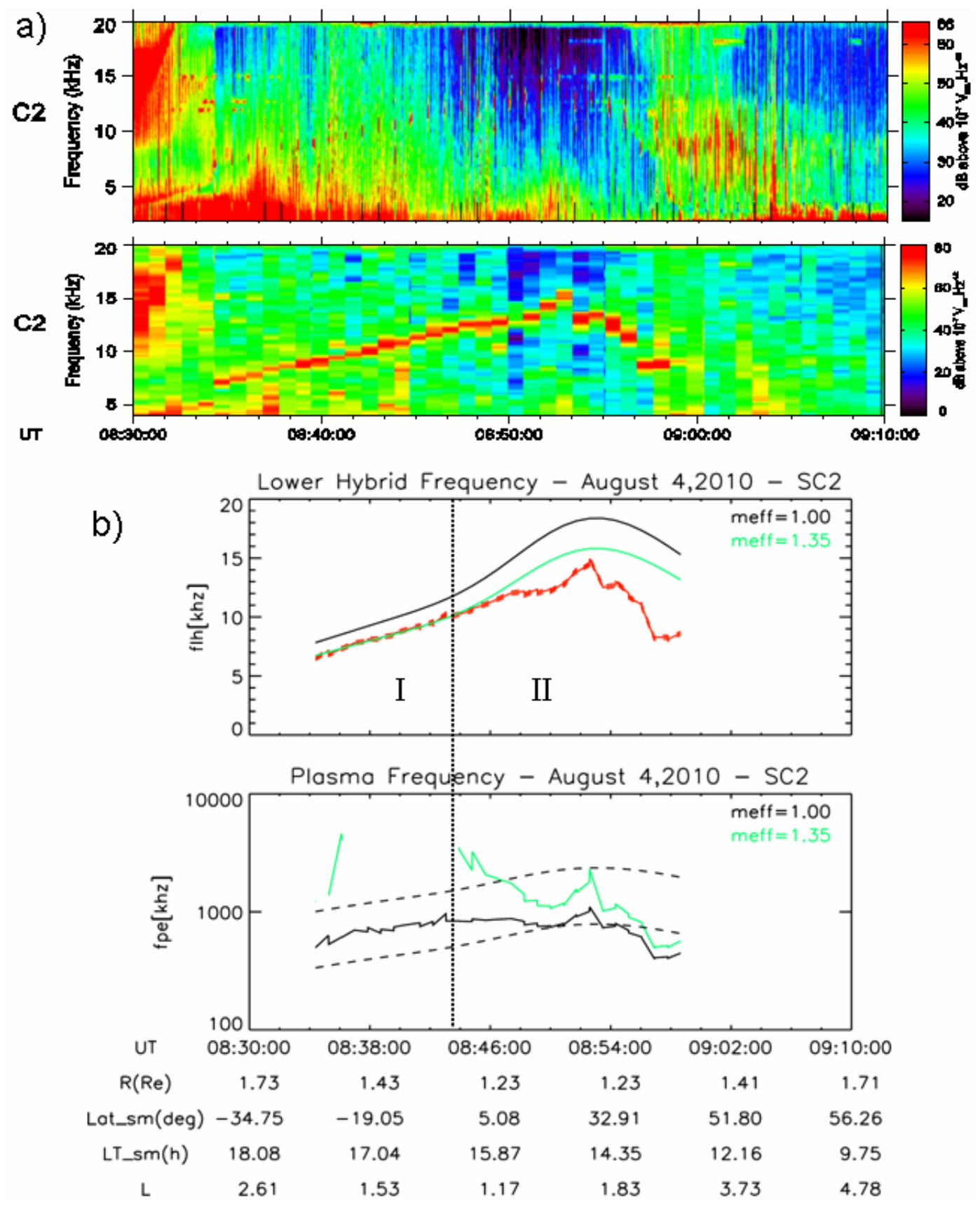

Fig. 6. Analysis of $F_{\mathrm{lh}}$ measurements on the $\mathrm{C} 2$ satellite, for the 4 August 2010 case event. (a) Frequency time spectrograms from the WHISPER instrument. Top: active and passive spectra. Bottom: active spectra. (b) Same parameters and presentation as panels (b) and (c) in Fig. 5.

that the effective ion mass is increasing with decreasing distance along the line of force (from a base level of $500 \mathrm{~km}$ ) which is the case here, from time interval I to time interval II, and in a more significant manner, during interval II (altitudes in the range $1600-1200 \mathrm{~km}$ ). If this is indeed the case, then the plasma frequency profile indicates a lower threshold of local electron density along the orbit. At this stage, we have no experimental basis to propose any estimate of the distance between the true plasma frequency and the one shown by the green line profile.

During the last selected time interval, from 18:13 to 18:17 UT, the satellite altitude is slowly increasing while L values encountered by the spacecraft are increasing more rapidly (see the lower panel of Fig. 7a). The effective mass is thus likely to decrease slowly, toward the value estimated in the first interval, since the satellite reaches altitudes covered 
a)
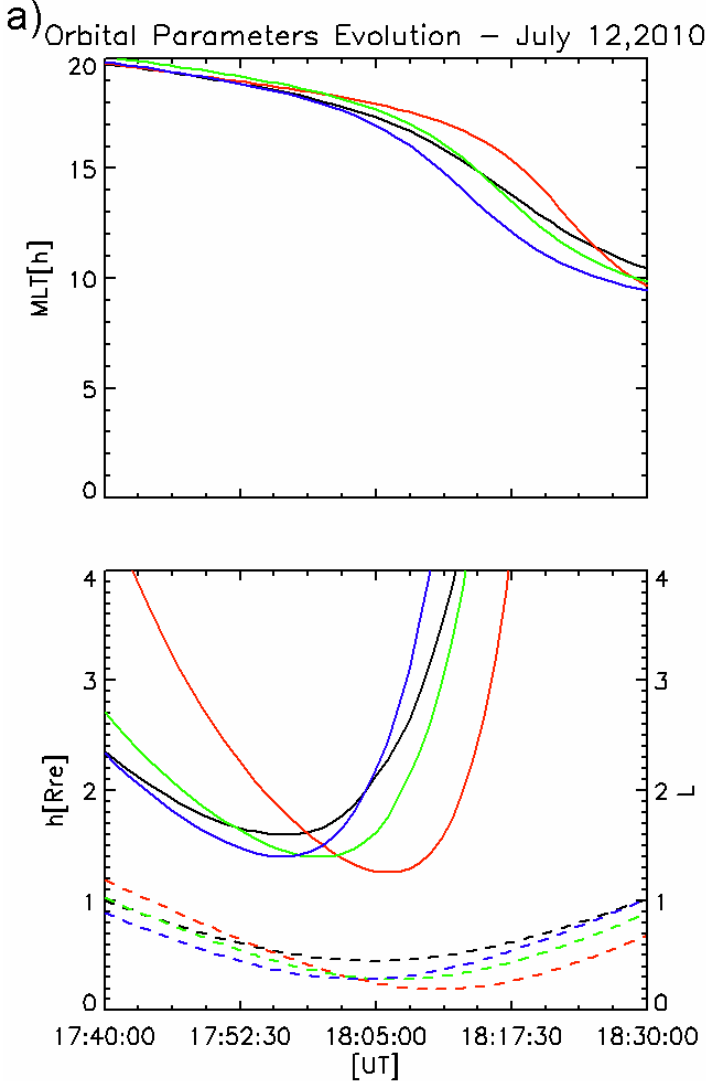

b)
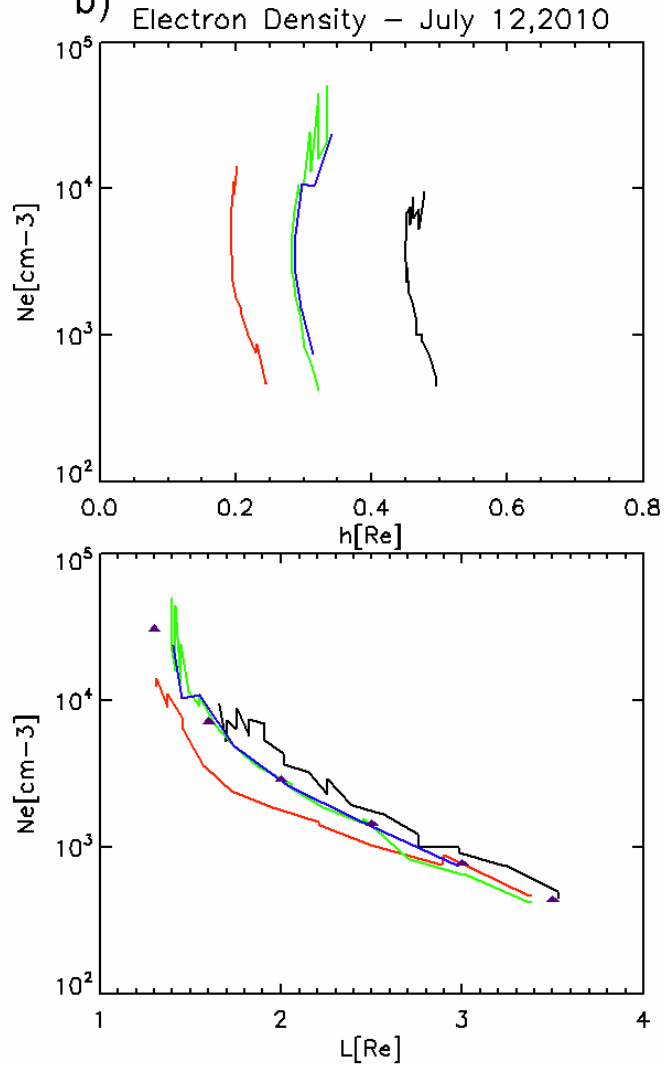

Fig. 7. (a) Evolution of orbital parameters, magnetic local time (upper panel) altitude (dashed lines) and L value (solid lines) (lower panel) for the event shown in Fig. 5. Colour codes according to satellite, black, red, green and blue respectively for C1, C2, C3 and C4. (b) Densities outbound of minimal $\mathrm{L}$ value encountered as function of altitude (top) and $\mathrm{L}$ value (bottom). $M_{\mathrm{eff}}$ values used in the calculations are deduced from the inbound $F_{\mathrm{lh}}$ measurements, $1.16,1.26,1.20$, and 1.18 for $\mathrm{C} 1, \mathrm{C} 2, \mathrm{C} 3$ and $\mathrm{C} 4$, respectively.

then $(>1600 \mathrm{~km})$ at the end of the sequence (after 18:16 UT). It is reasonable, on the other hand, to anticipate that, at the same time, the density is decreasing more rapidly, in inverse proportion to the volume of magnetic flux tubes encountered, such that the derived plasma-frequency profile shown by the green curve after 18:13 UT is governed mostly by density variations. Moreover, near the end of the sequence, it is probable that the inequality $F_{\mathrm{ce}} \gg F_{\mathrm{p}}$ is valid (see compared $F_{\mathrm{p}}$ and $F_{\mathrm{ce}}$ variations in Fig. 5c). In this case, Eq. (6) indicates that the electron density $N_{\mathrm{e}}$ (which varies as the square of $F_{\mathrm{p}}$ ) is proportional to $M_{\text {eff }}$. A bias introduced on $M_{\text {eff }}$ would be propagated on $N_{\mathrm{e}}$ by the same proportion. The error in the obtained $N_{\mathrm{e}}$ during this interval depends mainly on the quality of the guess about the actual $M_{\text {eff }}$ value; the uncertainty in $F_{\mathrm{lh}}$ measurements $( \pm 160 \mathrm{~Hz})$ being probably negligible.

Figure 6 presents WHISPER waves observations near the $F_{\mathrm{lh}}$ resonance for another event, 4 August 2010. In this case, the geomagnetic activity is higher ( $\mathrm{Kp}$ index equal to $3-$ ) than during the event of Fig. 3 (Kp index $0+$ to 1 ). The orbit is also slightly different (manoeuvres had taken place in between the two events, in order to rearrange the configuration of the CLUSTER constellation), but the main charac- teristics are very similar, with minimal $\mathrm{L}$ value encountered a few minutes before perigee. The top panels display frequency time spectrograms in a similar presentation to that in Fig. 2c. The upper one combines spectra obtained during sounding and passive operations, respectively, and the lower one displays the same active spectra as shown above, where pixels are expanded along the time scale for better visibility. Successive triggered $F_{\text {lh }}$ resonances appear clearly between 08:34 and 08:59 UT. No clear spectral signature of the $F_{\mathrm{lh}}$ resonance appear in the natural emissions, except between 08:56 and 08:59 UT, where bands of natural emissions display a lower frequency cut off at the $F_{\mathrm{lh}}$ resonance. Another banded emission with a clear lower frequency cut off is visible before 08:34 UT, increasing from 4 to $6 \mathrm{kHz}$. It is actually associated with resonances triggered by the sounder in its lowest frequency range $(<5 \mathrm{kHz})$. Difficult to distinguish in the spectrogram, they are seen when examining individual spectra (not shown). It appears that there is an abrupt change in the plasma regime near 08:34 UT (banded emissions disappear, and $F_{\mathrm{lh}}$ steps upward), which will not be discussed in this paper. 
Figure $6 \mathrm{~b}$ presents two panels similar to those shown in Fig. $5 \mathrm{~b}$ and c. The upper panel displays the measured $F_{\mathrm{lh}}$ and $F_{\mathrm{lh} 0}$ variations with time, together with the best fit of $F_{\mathrm{lh}}$ under assumption of infinite density, yielding an $M_{\text {eff }}$ value of 1.35. The lower panel displays $F_{\mathrm{ce}}$ variations (dashed line) and $F_{\mathrm{p}}$ variations (black and green lines) for $M_{\text {eff }}$ values equal to 1 and 1.35, respectively. For this event, the time interval where $F_{\mathrm{lh}}$ measurements are available can be split in two main sequences, marked I and II in Fig. 6b. From 08:34 to 08:44 UT, $F_{\mathrm{lh}}$ variations can be modelled by those occurring in a plasma regime where $F_{\mathrm{p}} \gg F_{\mathrm{ce}}$. The deduced effective mass, $M_{\text {eff }}=1.35$, is higher than for the 12 July event. This could be due to the higher geomagnetic activity, since the relative abundance of heavy ions inside the inner plasmaphere is increasing with activity (Berube at al., 2005). It could also result from different conditions at the magnetic foot points: both are sunlit during the $4 \mathrm{Au}-$ gust event (MLT 16.6), while they are placed near the terminator on 12 July (MLT 18.5). The high altitude ionosphere displays indeed higher concentration of heavy ions at the dayside than at night (Lemaire and Gringauz, 1998; Reynolds et al., 2001). The $L$ and altitude intervals swept when the above $M_{\text {eff }}$ estimate is qualified are $1.19<L<2$ and $1800 \mathrm{~km}<h<3600 \mathrm{~km}$, respectively. From 08:44 UT to 09:58 UT, the $F_{\mathrm{lh}} / F_{\mathrm{lh} 0}$ ratio goes down, and $F_{\mathrm{p}}$ variations are calculated, as a first approximation, by using the $M_{\text {eff }}$ value estimated before. The $F_{\mathrm{p}}$ profile derived is indicative of a lower limit of true plasma frequencies, since the altitudes explored are all smaller than during the first time interval (thus $M_{\text {eff }}$ likely underestimated). As with the July 12 th event, $F_{\mathrm{p}}$ decreases globally with increasing $\mathrm{L}$ values, but the detailed profile displays significant irregularities, in that case near perigee (altitudes near $1400 \mathrm{~km}$ ), at low L values $(L<2)$ and in the post noon sector. Irregular $F_{\mathrm{lh}}$ variations are also observed by other spacecraft that day, as well as in other case events, always in plasma regimes where it is not possible to disentangle density and $M_{\text {eff }}$ variations. These observations are currently under investigation.

In summary, $F_{\mathrm{lh}}$ measured along the CLUSTER orbit near perigee leads, via a reasonably simple strategy, to an estimate of the effective ion mass, and to useful information about plasma density (a lower threshold value and local fluctuations). The results obtained from two different case events indicate that those parameters can vary significantly with geomagnetic activity and/or MLT, as expected. The next section is a quantitative presentation of the density variations observed during the 12 July event, from the four different CLUSTER platforms.

\section{Density profiles versus $L$}

The evolution with time of the four CLUSTER satellite positions is indicated in Fig. 7a. The top panel shows MLT variations with time and the bottom panel provides $\mathrm{L}$ value (solid lines) and altitude (dashed lines) variations with time. The colour code is standard, respectively black, red, green and blue for $\mathrm{C} 1, \mathrm{C} 2, \mathrm{C} 3$ and $\mathrm{C} 4$. Analysis of the $F_{\mathrm{lh}}$ variations along the orbit leads to the following estimates: $M_{\text {eff }}=1.09$ for satellite $\mathrm{C} 1$, (highest altitudes), $M_{\mathrm{eff}}=1.26$ for $\mathrm{C} 2$ (lowest altitudes) and $M_{\text {eff }}=1.20$ and 1.18 respectively for C3 and $\mathrm{C} 4$ (intermediate altitudes). $M_{\mathrm{eff}}$ values are thus ordered as expected with altitudes, considering that the proportion of oxygen in the ionosphere decreases with altitude. Figure $7 \mathrm{~b}$ displays the density estimated from the $F_{\mathrm{lh}}$ measurements after crossing the innermost $\mathrm{L}$ shell, plotted as a function of the $\mathrm{L}$ parameter in the lower panel, and as a function of altitude in the upper panel. It is clear that the controlling parameter is $L$ : the four density profiles versus $L$ are close to each other, whereas the four density profiles versus altitude are unrelated and local time (not shown) does not seem to play a significant role (although it is difficult to disentangle local time and $L$ variations). In order to check that the order of magnitude of density values versus $L$ is realistic, we plot density values measured on $\mathrm{C} 3$ and $\mathrm{C} 4$ (triangles in deep purple), which are placed in between values seen on $\mathrm{C} 2$ and $\mathrm{C} 1$. The $\mathrm{C} 3$ and $\mathrm{C} 4$ values are reported in Fig. 8, adapted from Pierrard and Stegen (2008), showing densities derived from various empirical models. Orders of magnitude are clearly compatible. It is interesting to note that the differences noted by comparing the four profiles versus $L$ (bottom panel of Fig. 7b) are ordered according to altitude in an unexpected manner, i.e. as if densities were decreasing with altitudes (shown in top panel of Fig. 7b). Also remarkable is that the largest splits between the different density profiles versus $L$ occur for $\mathrm{L}$ values smaller than 2.5. In the case of satellite $\mathrm{C} 2$, this corresponds to the time interval noted as II in Fig. 5, when altitudes are decreasing and $M_{\text {eff }}$ is probably underestimated, leading to underestimated densities. This interpretation allows, by letting $M_{\text {eff }}$ vary in the expected direction, to recover a rational behaviour, where all density profiles are grouped toward the same one. Moreover, following further that line of interpretation (where $M_{\text {eff }}$ is underestimated near perigee), it is possible to obtain a rough estimate of the actual $M_{\text {eff }}$ value near the $\mathrm{C} 2$ perigee altitudes for that particular event, by assuming that the true density is close to the one indicated by the C3-C4 profile (triangles). This leads to $M_{\text {eff }} \sim 1.6$, instead of $M_{\text {eff }}=1.26$, which is reasonable for the altitudes considered $(1250 \mathrm{~km})$. This example illustrates qualitatively how the multi-point $F_{\mathrm{lh}}$ measurements could be used to form an optimized diagnostic about density and composition in the region explored by the CLUSTER constellation. A quantitative study would need the use of a common model, or of pertinent assumptions, constrained by actual measurements.

\section{Summary and conclusion}

This work presents a short review of the WHISPER relaxation sounder capabilities regarding plasma diagnostics in 


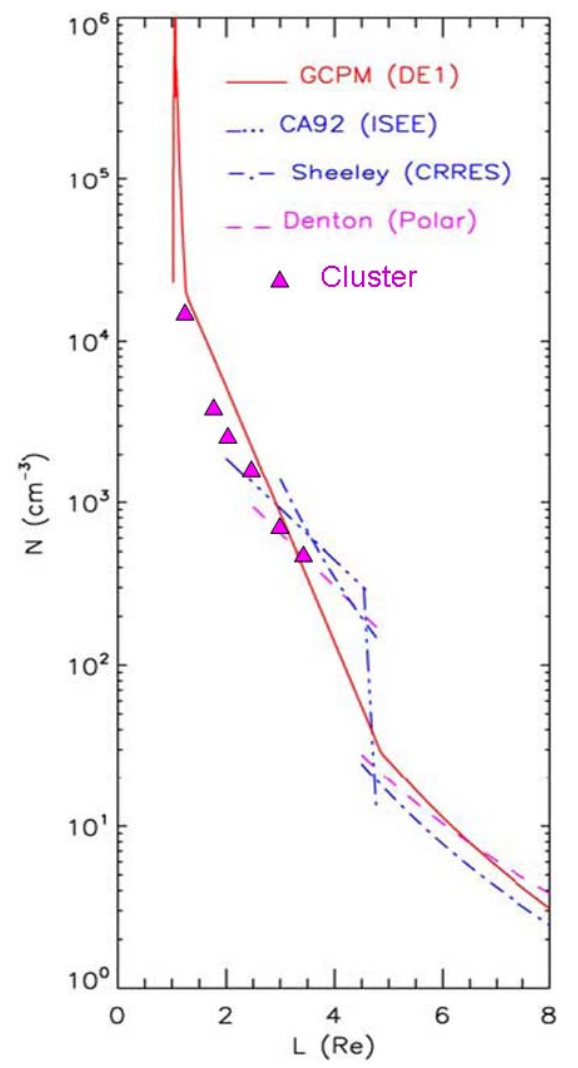

Fig. 8. Plasma density variation with $\mathrm{L}$ values. Estimates from $F_{\text {lh }}$ measurements on Cluster (values chosen shown in Fig. 7b), compared to empirical models (adapted from Pierrard and Stegen, 2008).

the plasmaphere region crossed by CLUSTER. Classically, the diagnostic is based on the observation of the upper hybrid resonance and other resonances in the frequency range around it. The quality of the diagnostics and information returned depends on which part of the plasmasphere body is crossed, and how. We show examples of spectrograms obtained during typical plasmasphere crossings at different phases of the CLUSTER mission. Lately (2009 onwards), another diagnostic approach has become feasible: observations of the lower hybrid resonances triggered by the relaxation sounder. This happens when CLUSTER is placed in the innermost plasmasphere ( $L=1.5-3.4)$ at low altitude $(0.2-$ $\left.0.7 R_{\mathrm{E}}\right)$. This paper describes which piece of information can be obtained from the lower hybrid resonance frequencies $F_{\mathrm{lh}}$, and how it is derived. The local $F_{\mathrm{lh}}$ frequency is always smaller than the frequency $F_{\mathrm{lh} 0}=F_{\mathrm{ce}} \sqrt{m_{\mathrm{e}} / m_{\mathrm{p}}}$ which is known with a good accuracy from magnetic field models or onboard measurements. By comparing the evolution of the ratio $F_{\mathrm{lh} 0} / F_{\mathrm{lh}}$ along the orbit, we estimate two key quantities, each valid for one of the two successive orbit elements, the effective mass $M_{\text {eff }}$, indicative of ion composition, and the electron density $N_{\mathrm{e}}$ (or rather a lower thresh- old estimate, and its fluctuations), respectively. The simple approach described provides $M_{\text {eff }}$ values in regions of small volume, i.e. along orbit elements of order $0.1 R_{\mathrm{E}}$ (hence more locally than $M_{\text {eff }}$ derived from ULF studies). The two case events analysed indicate that the quantities $M_{\text {eff }}$ and $\mathrm{Ne}$, although of expected order of magnitude, are varying significantly from one satellite of the constellation to another, and from one event to another. Moreover, some density profiles along the orbit reveal the presence of significant irregularities in the core of the plasmasphere $(L<2)$. We show, from the case event selected during low magnetic activity, that the effective ion mass is mainly controlled by altitude, and that the main parameter controlling the density is the $\mathrm{L}$ shell value. A qualitative illustration of how simultaneous measurements can be used to improve the quality of density estimation is proposed. Hence, the CLUSTER late mission phase offers a novel data basis, to investigate how other parameters such as local time, and geomagnetic activity, are controlling the plasma regime. No detailed evaluation of the characteristics of this data basis is available yet, but we can provide a few pieces of information: (i) starting June 2009 and prior to summer 2011, about 175 perigee passes show $F_{\text {lh }}$ resonances, with nearly all perigees being placed in the dayside sector (no data are available during the long eclipse seasons), with a reasonable sampling of MLT values; (ii) about half of those perigee passes are providing simultaneous $F_{\mathrm{lh}}$ observations, from two or more CLUSTER spacecraft; (iii) the latitudinal range explored is mostly medium latitudes in the Northern Hemisphere, including at times the equatorial region; (iv) altitudes and $\mathrm{L}$ values sampled are changing along the mission phase and with manoeuvres; several configurations of the constellation, with various satellites separations, are available. At the time of this writing, a significant effort has yet to be made in order to optimize the use of simultaneous $F_{\mathrm{lh}}$ measurements (thus under specific geophysical activity conditions) toward quantitative precise local density measurements. Finally, reliable measurement of the local $F_{\mathrm{lh}}$ is also of value toward a better interpretation of the many waves propagating inside the plasmaphere. This allows for instance to distinguish between boundaries remotely sensed or local. We show in one case event (4 August 2011) an example of waves of natural origin whose lower cut off frequency coincides with the local $F_{\mathrm{lh}}$ frequency. Such whistler waves could have experienced a cut off at a distance from their observation (Shklyar et al., 2010). We think that it is important to have access to a view as clear and as detailed as possible of the innermost plasmaphere, toward a better understanding and interpretation of the physics taking place there.

Acknowledgements. We thank the technical teams, at ESOC, ESTEC, and in LPC2E, for their great efforts, leading to the acquisition of an important scientific data basis. CNES and ESA funds supported WHISPER instrument construction and support WHISPER data analysis. JSP acknowledges support from NASA Goddard Space Flight Center under Grant NNX11AB38G. 
Guest Editor M. Taylor thanks B. Benson and V. Sonwalkar for their help in evaluating this paper.

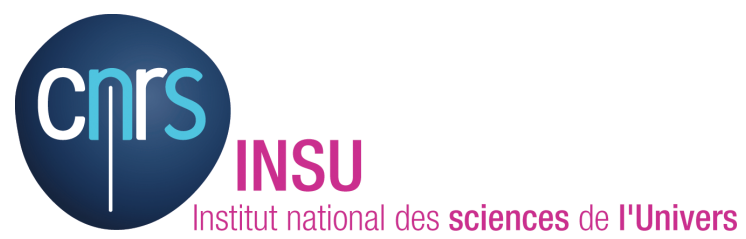

The publication of this article is financed by CNRS-INSU.

\section{References}

Angerami, J. J. and Thomas, J. O.: Studies of planetary atmospheres 1. The distribution of electrons and ions in the Earth's exosphere, J. Geophys. Res., 69, 4537-4560, 1964.

Balogh, A., Dunlop, M. W., Cowley, S. W. H., Southwood, D. J., Thomlinson, J. G., Glassmeier, K. H., Musmann, G., Lühr, H., Buchert, S., Acuña, M. H., Fairfield, D. H., Slavin, J. A., Riedler, W., Schwingenschuh, K., Kivelson, M. G., and the Cluster magnetometer team: The Cluster magnetic field investigation, Space Sci. Rev., 79, 65-91, 1997.

Barrington, R. E., Belrose, J. S., and Nelms, G. L.: Ion composition and temperature at $1000 \mathrm{~km}$ as deduced from simultaneous observations of a VLF plasma resonance and topside sounding data from the Alouette I satellite, J. Geophys. Res., 70, 1647-1664, 1965.

Béghin, C. and Debrie, R.: Characteristics of the electric field far from and close to a radiating antenna around the lower hybrid resonance in the ionospheric plasma, J. Plasma Physics, 8, 287310, 1972.

Bell, T. F., Inan, U. S., Sonwalkar, V. S., and Helliwell, R. A.: DE1 observations of lower hybrid waves excited by VLF whistler mode waves, Geophys. Res. Lett., 18, 393-396, 1991.

Berube, D., Moldwin, M. B., Fung, S. F., and Green, J. L.: A plasmapsheric mass density model and constraints on its heavy ion concentration, J. Geophys. Res., 110, A04212, doi:10.1029/2004JA010684, 2005.

Brice, N. M. and Smith, R. L.: Recordings from satellite Alouette I, A very-low-frequency plasma resonance, Nature, 203, 926, 1964.

Brice, N. and Smith, R. L.: Lower hybrid resonance emissions, J. Geophys. Res., 70, 71-80, 1965.

Brice, N. M., Smith, R. L., Belrose, J. S., and Barrington, R. E.: Recordings from satellite Alouette I, Triggered very-lowfrequency plasma emissions, Nature, 203, 926-927, 1964.

Carpenter, D. L. and Anderson, R. R.: An ISEE/Whistler model of equatorial electron density in the magnetosphere, J. Geophys. Res., 97, 1097-1108, 1992.

Carpenter, D. L. and Lemaire, J.: The Plasmasphere Boundary Layer, Ann. Geophys., 22, 4291-4298, doi:10.5194/angeo-224291-2004, 2004.

Craven, P. D., Gallagher, D. L., and Comfort, R. H.: Relative concentration of $\mathrm{He}+$ in the inner magnetosphere as observed by the DE 1 retarding ion mass spectrometer, J. Geophys. Res., 102, 2279-2289, 1997.

Darrouzet, F., De Keyser, J., Décréau, P. M. E., Gallagher, D. L., Pierrard, V., Lemaire, J. F., Sandel, B. R., Dandouras, I., Matsui,
H., Dunlop, M., Cabrera, J., Masson, A., Canu, P., Trotignon, J. G., Rauch, J. L., and André, M.: Analysis of plasmaspheric plumes: CLUSTER and IMAGE observations, Ann. Geophys., 24, 1737-1758, doi:10.5194/angeo-24-1737-2006, 2006.

Darrouzet, F., De Keyser, J., and Pierrard, V. (Eds.): The Earth's plasmasphere, a CLUSTER and IMAGE perspective, Springer, doi:10.1007/978-1-4419-1323-4, previously published in Space Sci. Rev., 145, 2009a.

Darrouzet, F., Gallagher, D. L., André, N., Carpenter, D. L., Dandouras, I., Décréau, P. M. E., De Keyser, J., Denton, R. E., Foster, J. C., Goldstein, J., Moldwin, M. B., Reinisch, B. W., Sandel, B. R., and Tu, J.: Plasmaspheric Density Structures and Dynamics: Properties Observed by the CLUSTER and IMAGE Missions, Space Sci. Rev., 145, doi:10.1007/s11214-008-9438-9, 2009 b.

Décréau, P. M. E., Fergeau, P., Krasnosel'skikh, V., Lévêque, M., Martin, P., Randriamboarison, O., Sené, F. X., Trotignon, J. G., Canu, P., Mögensen, P. B., and WHISPER Investigators: WHISPER, a resonance sounder and wave analyser: performances and perspectives for the CLUSTER misssion, Space Sci. Rev., 79, 157-193, 1997.

Décréau, P. M. E., Fergeau, P., Krasnoselskikh, V., Le Guirriec, E., Lévêque, M., Martin, Ph., Randriamboarison, O., Rauch, J. L., Sené, F. X., Séran, H. C., Trotignon, J. G., Canu, P., Cornilleau, N., de Féraudy, H., Alleyne, H., Yearby, K., Mögensen, P. B., Gustafsson, G., André, M., Gurnett, D. C., Darrouzet, F., Lemaire, J., Harvey, C. C., Travnicek, P., and Whisper experimenters: Early results from the Whisper instrument on Cluster: an overview, Ann. Geophys., 19, 1241-1258, doi:10.5194/angeo-19-1241-2001, 2001.

Décréau, P. M. E., Le Guirriec, E., Rauch, J. L., Trotignon, J. G., Canu, P., Darrouzet, F., Lemaire, J., Masson, A., Sedgemore, F., and André, M.: Density irregularities in the plasmasphere boundary Layer: CLUSTER observations in the dusk sector, Adv. Space Res., 36, 1964-1969, doi:10.1016/j.asr.2005.08.050, 2005.

El-Lemdani Mazouz, F., Rauch, J.-L., Décréau, P. M. E., Trotignon, J.-G., Vallières, X., Darrouzet, F., Canu, P., and Suraud, X.: Wave emissions at half gyroharmonics in the plasmasphere region: CLUSTER observations and statistics, Adv. Space Res., 43, 253-264, doi:10.1016/j.asr.2008.06.007, 2009.

Escoubet, C. P., Schmidt, R., and Goldstein, M. L.: CLUSTER - Science and mission overview, Space Sci. Rev., 79, 11-32, doi:10.1023/A:1004923124586, 1997.

Etcheto, J. and Bloch, J. J.: Plasma density measurements from the GEOS-1 relaxation sounder, Space Sci. Rev., 22, 597-610, 1978.

Gallagher, D. L., Craven, P. D., and Comfort, R. H.: Global core plasma model, J. Geophys. Res., 104, 18819-18833, 2000.

Gustafsson, G., Boström, R., Holback, B., Holmgren, G., Lundgren, A., Stasiewicz, K., Ahlen, L., Mozer, F. S., Pankow, D., Harvey, P., Berg, P., Ulrich, R., Pedersen, A., Schmidt, R., Butler, A., Fransen, A. W. C., Klinge, D., Thomsen, M., Fâlthammar, C. G., Lindqvist, P.-A., Christenson, S., Holtet, J., Lybekk, B., Sten, T. A., Tanskanen, P., Lappalainen, K., and Wygant, J.: The electric field and wave experiment for the Cluster mission, Space Sci. Rev., 79, 137-156, 1997.

Hamelin, M.: Excitation of LHR electrostatic waves in a warm magnetoplasma by current pulses: Application to the VLF echoes excited by the Isis HF transmitter, Radio Sci., 15, 49-57, 1980. 
Johnstone, A. D., Alsop, C., Burge, S., Carter, P. J., Coates, A. J., Coker, A. J., Fazakerley, A. N., Grande, M., Gowen, R. A., Gurgiolo, C., Hancock, B. K., Narheim, B., Preece, A., Sheather, P. H., Winningham, J. D., and Woodliffe, R. D.: PEACE: a plasma electron and current experiment, Space Sci. Rev., 79, 351-398, 1997.

Lemaire, J. F. and Gringauz, K. I.: The Earth's Plasmasphere, Cambridge University Press, New York, 1998.

Macmillan, S., Maus, S., Bondar, T., Chambodut, A., Golovkov, V., Holme, R., Langlais, B., Lesur, V., Lowes, F., LÙhr, H., Mai, W., Mandea, M., Olsen, N., Rother, M., Sabaka, T., Thomson, A., and Wardinski, I.: Ninth Generation International Geomagnetic Reference Field Released, EOS Transactions, AGU, 84, 503503, 2003 and Geophys. J. Int., 155, 1051-1056, 2003.

Masson, A., Santolik, O., Carpenter, D. L., Darrouzet, F., Décréau, P. M. E., Green, J. L., Grimald, S., El-Lemdani Mazouz, F., Moldwin, M. B., Nemec, F., and Sonwalkar, V. S.: Advances in plasmaspheric wave research with CLUSTER and IMAGE observations, Space Sci. Rev., 145, 137-191, doi:10.1007/s11214009-9508-7, 2009.

Moullard, O., Masson, A., Laakso, H., Parrot, M., Décréau, P. M. E., Santolik, O., and André, M.: Density modulated whistler mode emissions observed near the plasmapause, Geosphys. Res. Lett., 29, 1975, doi:10.1029/2002GL015101, 2002.

Muldrew, D. B.: Electron resonances observed with topside sounders, Radio Sci., 7, 779-789, 1972.

Palmer, F. H. and Barrington, R. E.: excitation of io resonances by thebIsis 2 HF transmitter, J. Geophys. Res., 78, 8167-8179, 1973.

Pedersen, A., Cornilleau-Wherlin, N., De La Porte, B., Roux, A., Bouabdellah, A., Décréau, P. M. E., Lefeuvre, F., Sené, F. X., Gurnett, D., Huff, R., Gustafsson, G., Holmgren, G., Woolliscroft, L., St. C. Alleyne, H., Thompson, J. A., and Davies, P. N. H.: The Wave Experiment Consortium (WEC), Space Sci. Rev., 79, 157-193, 1997.

Pierrard, V. and Lemaire, J.: Exospheric model of the plasmasphere, J. Atmos. Solar-Terr. Phys., 63, 1261-1265, 2001.

Pierrard, V. and Stegen, K.: A three-dimensional dynamic kinetic model of the plasmasphere, J. Geophys. Res. 113, A102099, doi:10.1029/2008JA013060, 2008.

Rauch, J. L., Suraud, X., Décréau, P. M. E., Trotignon, J. G., Ledée, R., El-Lemdani Mazouz, F., Grimald, S., Bozan, G., Vallières, X., Canu, P., Darrouzet, F.: Automatic determination of the plasma frequency using image processing on whisper data. Proceedings of the Cluster and Double Star Symposium - 5th Anniversary of Cluster in Space, Noordwijk, The Netherlands, 1923 September 2005, ESA SP-598, January 2006.

Reinish, B. W., Haines, D. M., Bibl, K., Cheney, G., Galkin, I. A., Huang, X., Myers, S. H., Sales, G. S., Benson, R. F., Fung, S. F., Green, J. L., Taylor, W. W. L., Bougeret, J.-L., Manning, R., Meyer-Vernet, N., Montcuquet, M., Carpenter, D. L., Gallagher, D. L., and Reiff, P.: The Radio Plasma Imager Investigation on the IMAGE spacecraft, Space Sci. Rev., 91, 319-359, 2000.

Reynolds, M. A., Meléndez-Alvira, D. J., and Ganguli, G.: Equatorial coupling between the plasmasphere and the topside ionosphere, J. Atmos. Sol. Terr. Phys., 63, 1267-1273, 2001.
Shklyar, D. R., Parrot, M., Chum, J., Santolik, O., and Titiva, E. E.: On the orogin of lower- and upper- frequency cutoffs on wedge - like spectrograms observed by DEMETER in the midlatitude ionosphere, J. Geophys. Res., 115, A05203, doi:10.1029/2009JA014672, 2010.

Smith, R. L. and Brice, N. M.: Propagation in multicomponent plasmas, J. Geophys. Res., 69, 5029-5040, 1964.

Sonwalkar, V. S., Reddy, A., Carpenter, D. L., and Reinisch, B. W.: Whistler mode sounding of the magnetosphere: Measurement of electron density, ion composition $(\mathrm{H}+, \mathrm{HE}+, \mathrm{O}+)$, and density irregularities along the geomagnetic field, Commission G XXIXth URSI General Assembly, Chicago, 7-16 August 2008.

Sonwalkar, V. S., Carpenter, D. L., Reddy, A., Proddaturi, R. K., Hazra, S., Mayank, K., and Reinisch, B. W.: Magnetospherically reflected, specularly reflected, and backscattered whistler mode radio-sounder echoes observed on the IMAGE satellite, Part 1: Observations and Interpretation, J. Geophys. Res., accepted, doi:10.1029/2011JA016759, 2011a.

Sonwalkar, V. S., Reddy, A., and Carpenter, D. L.: Magnetospherically reflected, specularly reflected, and backscattered whistler mode radio-sounder echoes observed on the IMAGE satellite, Part 2: sounding of electron density, ion effective mass (meff), ion composition $(\mathrm{H}+, \mathrm{He}+, \mathrm{O}+)$, and density irregularities along the geomagnetic field line, J. Geophys. Res., accepted, doi:10.1029/2011JA016760, 2011b.

Trotignon, J. G., Etcheto, J., and Thouvenin, J. P.: Automatic determination of the electron density measured by the relaxation sounder on-board ISEE 1, J. Geophys. Res., 91, 4302-4320, 1986.

Trotignon, J. G., Décréau, P. M. E., Rauch, J. L., Randriamboarison, O., Krasnoselskikh, V., Canu, P., Alleyne, H., Yearby, K., Le Guirriec, E., Séran, H. C., Sené, F. X., Martin, Ph., Lévêque, M., and Fergeau, P.: How to determine the thermal electron density and the magnetic field strength from the Cluster/Whisper observations around the Earth, Ann. Geophys., 19, 1711-1720, doi:10.5194/angeo-19-1711-2001, 2001.

Trotignon, J. G., Décréau, P. M. E., Rauch, J. L., Le Guirriec, E., Canu, P., and Darrouzet, F.: The Whisper relaxation sounder onboard Cluster: a powerful tool for space plasma diagnosis around the Earth, Cosmic Research, 41, 345-348, 2003, translated from Kosmicheskie Issledovaniya, 41, 369-372, 2003.

Trotignon, J.-G., Décréau, P. M. E., Rauch, J. L., Vallières, X., Rochel, A., Kougblénou, S., Lointier, G., Facskó, G., Canu, P., Darrouzet, F., and Masson, A.: The whisper relaxation sounder and the cluster active archive. The Cluster Active Archive, Studying the Earth's Space Plasma Environment, edited by: Laakso, H., Taylor, M. G. T. T., and Escoubet, C. P., Astrophysics and Space Science Proceedings, 185-208, Berlin: Springer, 2010.

Tsyganenko, N. A. and Sitnov, M. I.: Modeling the dynamics of the inner magnetosphere during strong geomagnetic storms, J. Geophys. Res., 110, A03208, doi:10.1029/2004JA010798, 2005. Woolliscroft, L. J. C., Alleyne, H. ST. C., Dunford, C. M., Sumner, A., Thompson, J. A., Walker, S. N., Yearby, K. H., Buckley, A., Chapman, S., Gough, P., and the DWP investigators: The Digital Wave-Processing experiment on Cluster, Space Sci. Rev., 79, 209-231, 1997. 